Elsevier required licence: () 2019

This manuscript version is made available under the CC-BY-NC-ND 4.0 license http://creativecommons.org/licenses/by-nc-nd/4.0/

The definitive publisher version is available online at https://doi.org/10.1016/j.ijepes.2019.05.045 


\title{
A Hybrid Prediction-Based Microgrid Energy Management Strategy Considering Demand-Side Response and Data Interruption
}

Dongmei Yuan ${ }^{1}$, Zhigang Lu ${ }^{*}$, Jiangfeng Zhang ${ }^{2}$, Xueping $\mathrm{Li}^{1}$

(*Corresponding author, zhglu@ysu.edu.cn)

\author{
1. Institute of Electrical Engineering, Yanshan University, Qinhuangdao 066004, P. R. China \\ 2. School of Electrical and Data Engineering, University of Technology Sydney, Ultimo, NSW 2007, Australia
}

\begin{abstract}
Data interruption may cause the centralized energy management system of a microgrid (MG) to collapse. To solve this problem, a hybrid prediction-based energy management strategy is proposed in this paper to predict interrupted data for the centralized dispatching process of an MG. This hybrid prediction method is designed following a combination of model predictive control and extreme learning machine techniques. Based on the predicted data of distributed energy resources and three types of loads from demand-side response, an optimization model is formulated to minimize the operational cost. If some predicted data are interrupted during transmission, then a prospect vulnerability assessment method is applied to select a neighboring device to predict the interrupted data. In the end, an improved particle swarm optimization algorithm is proposed with the help of genetic algorithms to accelerate convergence to global optimal solutions for the proposed MG energy management problem. The effectiveness of the proposed models and solution methods is also verified by a case study.
\end{abstract}

Keywords: data interruption; microgrid; hybrid prediction; demand-side response; energy management

\section{Nomenclature}

Abbreviation

MG

Microgrid

DER

Distributed energy resource

DSR

Demand-side response

IGA

Improved genetic algorithm

photovoltaic PV

ELM Extreme learning machine

MPC Model predictive control

PSO Particle swarm optimization

EMS Energy management system

WT Wind turbine

MT Micro turbine

FC Fuel cell

EMD Empirical mode decomposition

IMFs Intrinsic modal functions

MAPE Mean absolute percent error

CPN cyber-physical node

RMSE Root mean square error

Parameters

$U_{i} \angle \theta_{i}(K)$

$I_{l d i}, I_{l q i}$

$U_{D C i}$

$Z_{\text {inverteri }}$

$Z_{\text {Linei }}$

$\beta_{i}$
The obtained voltage in the voltage synthesis part

Obtained by Park transformation on the inductive current $I_{l i} \quad$ (including $I_{l a i}(k+1), \quad I_{l b i}(k+1)$, and $\left.I_{l c i}(k+1)\right)$

The voltage of the i-th DER

The impedance of the i-th inverter

The line impedance

The weight of the $i$-th hidden layer node to the output layer in ELM

The activation function (i.e., mapping function) from the input layer to the hid-

den layer in ELM

The weight for the input layer to the $i$-th hidden layer node in ELM

The degree of bias of the $i$-th hidden layer node in ELM

The output matrix of the hidden layer in

$\boldsymbol{H}$

$\boldsymbol{\beta}$

$c_{\mathrm{ELM}_{i}}(K)$

$y(K)$

$y_{\mathrm{ELM}_{i}}(K)$

$y_{\mathrm{MPC}}(K)$

$I_{\text {qrefi }}(K)$

$x_{\text {train } . i}$

$x_{\text {train } . i}^{\prime}$

$d_{L q(l)}$

$I_{1, L q(l)}, I_{2, L q(l)}$

$\lambda_{L q(l)}$

$C_{C 1 j}$

$C_{C 2 j}$

$C_{C 3 j}$

\section{ELM}

An $N$ dimensional vector consisting of weights of the hidden layer to the output layer in ELM

The weight of the $i$-th ELM at $K \mathrm{~T}$

The historical data at $k \mathrm{t}$

The output of the $i$-th elm at $k \mathrm{t}$

The output of MPC at kt

The number of historical data

The $i$ th historical data

The corresponding predicted value

The electrical length (i.e., line impedance magnitude) of the $l$-th branch

The importance degrees of the source and flow nodes in the $l$-th branch, respectively The utilization ratio of the $l$-th branch of $L_{q}$

The importance degree of the $j$-th cyber nod

The number of failures at the $j$-th node during the year

The protective measures of the $j$-th cyber node 
$C_{C i j}$

$\xi_{i j}$

$C_{i \min }, C_{i \max }$

$V_{j}$

$\pi_{i}^{+}$

$\pi_{i}^{-}$

$\Delta \xi_{i j}$

$\alpha$ and $\beta$

$\lambda$

$\gamma^{+}$or $\gamma^{-}$

$a, b, c$ and $d$

$P_{\text {DERg }}(t)$

$\delta_{\mathrm{DER} g}(t)$

$c_{\text {grid }}(t)$

$P_{\text {grid }}(t)$

$\lambda_{\text {bat }}$

$P_{\text {bat }}(t)$

$\varepsilon_{I l i}(t)$

$\rho_{I l i}(t)$

$P_{I l i}(t)$

$\lambda_{\text {bat }}$

$P_{\text {bat }}(t)$

$\varepsilon_{c l i}(t)$

$\rho_{c l i}(t)$
The value of the $i$-th index

The value of $C_{C i j}$ after treatment

The minimum and maximum values of the

i-th index, respectively

The prospect value of the $j$-th node

For the $i$-th index, the decision-making weights for decision maker who has profits

For the $i$-th index, the decision-making weights for decision makers who have losses

The difference between $\xi_{i j}$ and the selected reference value

Sensitivity coefficients ( $0<\alpha<1$, $0<\beta<1$ ). And in general, $\alpha=0.88$, $\beta=0.9$.

The loss avoidance coefficient

The attitude coefficients of income or loss, and they are usually selected as 0.61 .

Constant coefficients of generation costs

The output power of the $g$-th DER

The start and stop state variable of the $g$-th DER

The selling/buying price of electricity

The amount of power exchanged mg and main grid

The cost coefficient of energy storage unit

The corresponding active power of energy storage unit

The dispatching cost coefficient of interruptible load

The interruptible rate

The power of the $i$-th interruptible load

The cost coefficient of energy storage unit

The corresponding active power of energy storage unit

The dispatching cost coefficient of flexible load

The flexible rate

$$
P_{c l i}(t)
$$$$
S
$$

$I$

$J$

$P_{\mathrm{DER} g}^{\min }$ and $P_{\mathrm{DER} g}^{\max }$

$\Delta P_{\mathrm{DER} g}$

$T^{u p}$

$\tau_{1}, \tau_{2}$

$T^{o p t}$

$T^{\text {down }}$

$P_{n l k}(t)$

$\Omega$

$P_{\text {bat }}^{\min }(t)$

$P_{\text {bat }}^{\max }(t)$

$P_{\text {grid }}^{\max }$ and $P_{\text {grid }}^{\min }$

$\rho_{I l i}^{\max }$ and $\rho_{I l i}^{\min }$

$T_{f l j, s t a r t}$ and $T_{f l j . \text { end }}$

$\delta_{P_{f l j}}$

$T_{f l j}$

$P_{C R}^{k}\left(P_{M R}^{k}\right)$

$p_{C R}^{\max }$ and $p_{C R}^{\min }\left(p_{M R}^{\max }\right.$

and $p_{M R}^{\min }$ )

$\rho_{k}$

$n$
The power of the $j$-th flexible load

The total number of time samples

The number of controllable DERs

The number of interruptible loads

The number of flexible loads

The minimum and maximum power of the $g$-th DER, respectively

The ramp rate of the $g$-th DER

The minimum running time

Auxiliary variables

The selected optimal time period

The minimum downtime

The active power of the $k$-th uncontrollable load

The number of uncontrollable loads and

The allowable minimum and maximum values of power variation

The minimum and maximum allowed power exchange between MG and main grid

The maximum and minimum interruptible rate of interruptible load

The start and stop moment to adjust the $j$-th flexible load

The corresponding power variable of the $j$-th flexible load during $T_{f j \text {.start }} \sim T_{f j \text {.end }}$

The running time of the $j$-th flexible load

The selected crossover rate (mutation rate) at the $k$ th iteration

The maximum and minimum values of the crossover rate (mutation rate)

The aggregation degree of particles at the $k$ th iteration

The selected number of iteration

\section{Introduction}

With the possible depletion of fossil fuel energy, more and more distributed renewable energy generations have been integrated to the power grid through microgrids (MGs) [1]. An MG forms a bridge linking distributed energy resources (DERs) and distribution network, and it is mainly composed of distributed generation units, loads and energy storage systems [2]. Optimal energy management of DERs and efficient operation of MGs are important research top- 
ics at present $[3,4]$. Unlike conventional energy generations, renewable energy is intermittent and thus brings a great challenge to the reliable operation of MGs. In addition, during upstream failures, an MG could be automatically disconnected from the main grid and operate in island mode to satisfy its loads under distributed intelligent controls [5-7]. One of the key functionalities in MG's energy management strategies is optimal scheduling, which includes optimal unit commitment, dispatch of generation resources, and demand management of flexible loads [8].

The future MG will be restructured as a cyber-physical system which has components not only to carry power flow, but also to transmit data for advanced distributed control purpose [9]. There are mainly two approaches in energy management strategies: centralized and decentralized. A decentralized approach aims to optimize economical operation of an MG while providing the highest possible autonomy to different DERs and loads. Decentralized approach has been addressed in [10] by using the multi-agent system framework. A centralized approach is featured by a central controller that is provided with the relevant communication data about the MG for the purpose of determining the dispatch of the DERs according to selected objectives [11]. Based on these communication data, centralized energy scheduling strategy is widely used in MGs. A centralized strategy is suitable for a single MG, and requires less communication data to operate $[12,13]$. This is also the main type of scheduling strategies studied in this paper.

Demand-side response (DSR) is a popular technique in the energy management of MG. Its objective is to benefit both of the power consumers and power suppliers through reshaping the load curve with the help of flexible loads and interruptible loads. The end-users taking part in demand response shall be offered either price or service incentives. Moreover, combined operation of responsive energy storage systems with demand response will lead to greater reliability of energy storage systems in distribution network with intermittent generation sources. It can greatly increase the flexibility in addressing the power imbalance problem in MG [14, 15]. At present, there are many existing studied on DSR. For example, the influence of DSR on the UK economy and the roles of end users are discussed in [16], optimization approaches to the application of DSR in power systems are reviewed in [17], a general DSR application in industrial electric load is reported in [18], and a DSR-based energy management scheme is applied to MG in [19]. These studies show that DSR will play more and more important roles for the future power grid, especially MGs, since direct control on interruptible or flexible load is possible to reduce MG operational cost and increase system reliability Considering the carbon emission reduction problem, the day-ahead outputs of DERs' buying-back schemes are studied in [21], where convex optimization algorithms are used to maximize the system social welfare. Uncertainties from power supply and load demand are considered in DSR to minimize the MG system cost in [22]. In [23], a heuristic-based evolutionary algorithm is presented to solve the DSR problem by a day-ahead load shifting technique for a complicated MG, and substantial savings are achieved through reduced peak load. Reference [24] studies the reverse power flow problem from rooftop photovoltaic (PV) system to the substation which causes a voltage rise when generation is larger than the load, and a DSR system is proposed to shift the operation of flexible loads from peak consumption hours to high PV production periods. A real-time DSR with communication networks is studied in [25] and a solution is derived from game theory to smooth the peak-to-average ratio, where it is also assumed to reward customers for accurate load forecast. Based on game theory, a real-time distributed algorithm is proposed in [26] to minimize customer bills by reducing peak demand. All adjustable loads are controlled in [27] to shift energy consumption from one time period to another, and thus obtain reduced peak-demand and flattened load curve. The above literature study shows the increasingly important role of DSR in future power grid and in particular MGs, since the DSR of interruptible or flexible load can have significant impact to the reliable and economic operation of the MGs.

Although existing approaches are able to achieve satisfactory results for different objectives, there are still the following challenges. Firstly, the obtained optimization problems are solved by intelligent algorithms, which are often trapped at local optima and introduce undesired disturbances to the system for online implementation since they are based on stochastic search. Secondly, the whole dispatching process will collapse if the communication data is interrupted in centralized approaches, and this will lead to the collapse of the whole MG. Thirdly, energy scheduling controls are mainly based on prediction data, which will lead to wrong optimization schemes if the prediction accuracy is low.

To solve the above problems, a hybrid prediction-based energy management strategy is presented in this paper. The simplified process is as below: Based on the predicted data of DERs and three types of loads, an optimization model is formulated to minimize the operational cost under DSR. To solve the problem of data interruption, a neighbor prediction method based on prospect vulnerability assessment is designed. An improved particle swarm algorithm is also proposed to improve convergence speed. The main contribution is highlighted below:

- A hybrid prediction method which combines model predictive control (MPC) and multiple extreme learning machines (ELMs) is designed. This method combines the advantages of MPC and ELM, and thus has better prediction accuracy than using either MPC or ELM alone.

- A neighbor prediction method based on prospect vulnerability assessment is designed to solve the problem of data interruption within the MG energy optimization model. When the transmission of predicted data of some devices in the system is interrupted, the optimization model can still be formulated, and the corresponding dispatching strategy can also be obtained.

- An optimization model considering DSR is designed and a particle aggregation degree based particle swarm optimization (PSO) algorithm is proposed to solve this problem. Through this design, the whole operation of MG can be opti- 
mized effectively. The optimization problem computing speed is also improved.

The remaining parts of this paper are organized as follows. The structure of the MG and the energy management system are presented in Section II. In Section III, the hybrid predictive method is proposed. In Section IV, the interruption problem in data transmission is solved. The optimal models are constructed in Section V. Simulations for case studies are provided in Section VI to demonstrate the effectiveness of the proposed control strategy. Conclusions are drawn in Section VII.

\section{Structure of the energy management system}

Fig. 1 shows the energy management system (EMS) and the MG which consists of a wind turbine (WT), a PV system, a micro turbine (MT), an energy storage unit, a fuel cell (FC) and three different types of loads (interruptible load, flexible load and fixed load) [7]. In these loads, interruptible load is a load that can be removed directly from the system; flexible load is a load with adjustable usage time; and fixed load is a critical load that must be met as required. Because of the intermittent and uncertain characteristics of DERs (e.g., WT and PV), an energy management strategy is needed to solve the problem of potential data interruption.

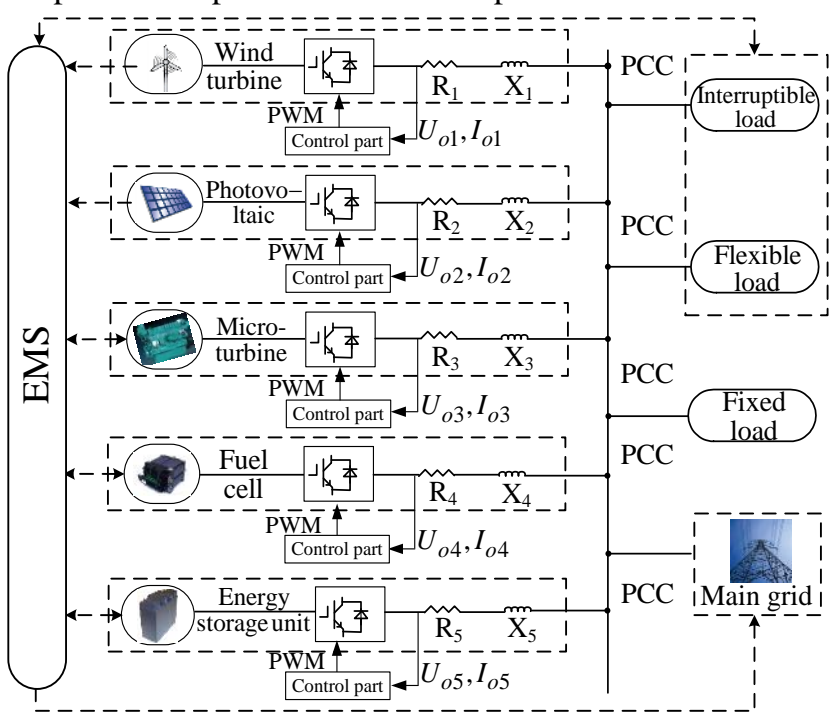

Fig. 1 The Micro-grid structure considering demand-side response

A simplified progress of rolling optimization is shown in Fig. 2. The optimization sampling period is one hour, while the overall optimization period is one day. Based on the current predicted data of DERs and loads, the corresponding optimization scheme is carried out to accomplish the energy management of MG. Afterwards, the needed data (i.e., power generation of controllable DERs, power exchanged with main grid, and the planned adjustable loads) can be obtained. Each controlled variable in the current sampling time period can be obtained by the predicted data in the previous sampling period, and this is why it is called rolling optimization. In other words, the dispatch scheme of current time period will affect the predicted data in the next period.

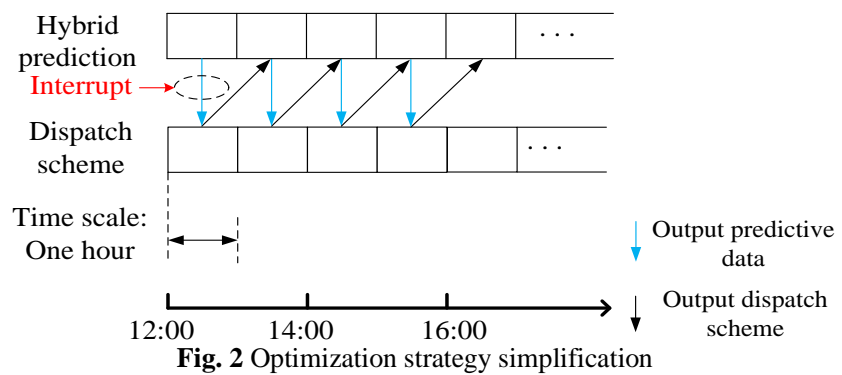

However, if the transmission of predicted data is interrupted, the dispatch progress will be interrupted. This will affect the operation of each DER, energy storage and the three types of loads in the following time periods. In serious cases, the whole system will crash. Therefore, for each DER (or energy storage unit), a neighbor predictive method based on cumulative prospective theory [28] and vulnerability assessment theory is proposed to solve this problem. This method relies on neighbor DER (or energy storage units).

The specific dispatch process of MG is shown in Fig. 3 , where the blue part represents the hybrid prediction part; the pink part represents the energy management part based on DSR; the grey part represents vulnerability assessment and neighbor prediction. The energy management processes are as follows: First of all, the predicted data from the hybrid prediction part in the uncontrollable DERs will be transmitted to the EMS via the sensors; then, the predicted data will be used to judge the current power balance between supply and demand. After deciding the power balance, the corresponding energy scheduling scheme will be used to control the operation of the whole MG. Meanwhile, the load information is also used for energy management at the next optimization iteration. In this way, a rolling optimization process is completed.

In addition, if the predicted data of the $i$-th uncontrollable DER is interrupted, the prospective vulnerability assessment and neighbor prediction are activated. That is, through vulnerability assessment, the neighbor DER with the lowest vulnerability is selected at first. The corresponding prediction and data transmission are completed by the selected DER instead of the $i$-th DER. In this way, the EMS can be maintained when there is data interruption. In addition, when the predicted data of the $i$-th DER is interrupted, the parts of prospect vulnerability assessment and neighbor prediction will be activated. Assume the data transmission of the $i$-th DER is interrupted, and the specific process is as below: Firstly, through the vulnerability assessment, the DER with the lowest vulnerability is selected in the neighbor DERs of the $i$-th DER. Then, the selected DER will be used to complete the corresponding prediction and data transmission. In this way, the normal operation of EMS can be ensured even if data interruption occurs in the $i$-th DER. 


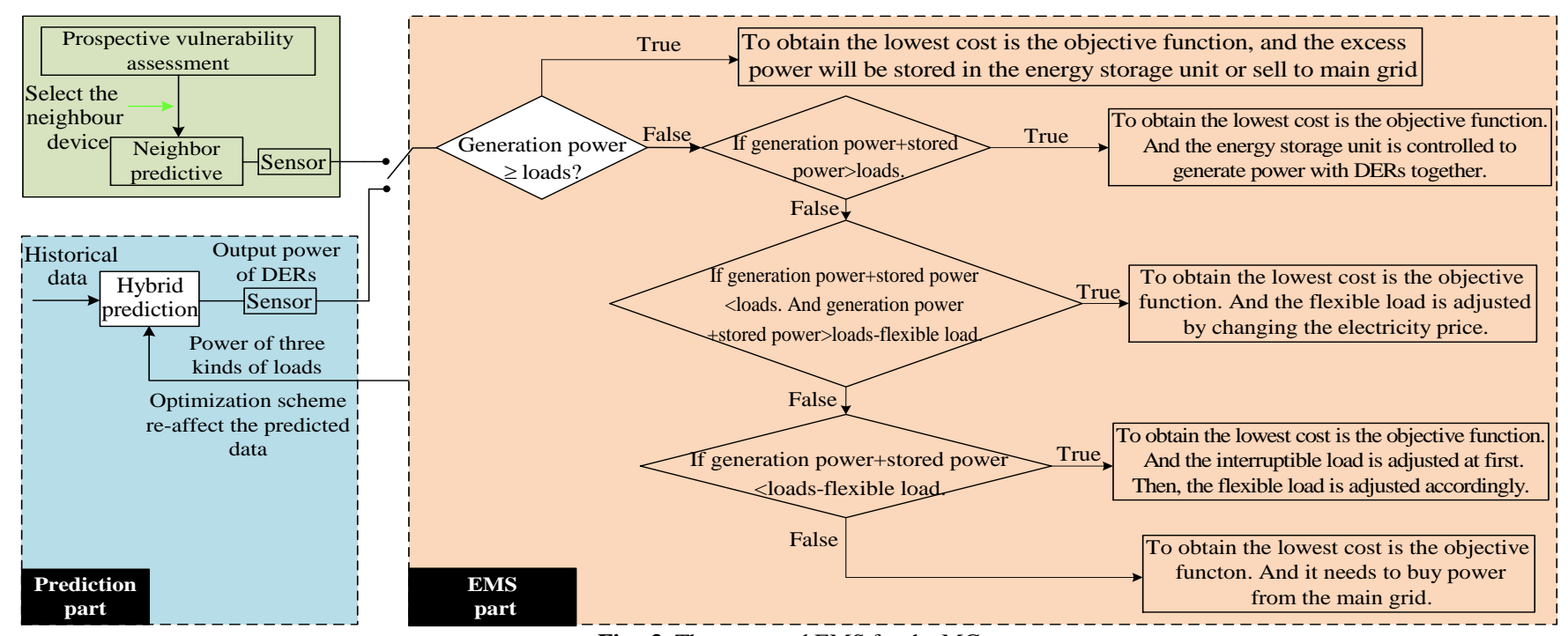

\section{The hybrid prediction method}

The inverter-based control structure of DERs can be simplified as Fig. 4, which will be explained in Subsection III.1. Based on this structure, MPC can be used for data prediction. Although MPC is robust, a hybrid predictive method combining ELM and MPC is designed in order to improve the real-time prediction performance. Considering different structures of the three types of loads, multiple weighted ELMs are used in the hybrid prediction. Note that WT and PV generation are affected by wind speed, solar irradiance and other factors, which result in power fluctuation and make the prediction more difficult. To solve this problem, the empirical mode decomposition (EMD) is used to pre-process historical data before using multiple ELMs for the power generation prediction from WT and PV [29], where the key idea is to decompose historical data with high volatility and long time durations into small groups of data with low volatility and shorter time durations. This greatly reduces the forecasting difficulty. Detailed design of this hybrid prediction process is given as follows.

\section{1 Application of MPC}

The inverter-based control structure of DER in Fig. 1 is shown as Fig. 4. Based on the inverter control structure, MPC can be applied to predict the active power output of DER. The detailed steps are given as follows.

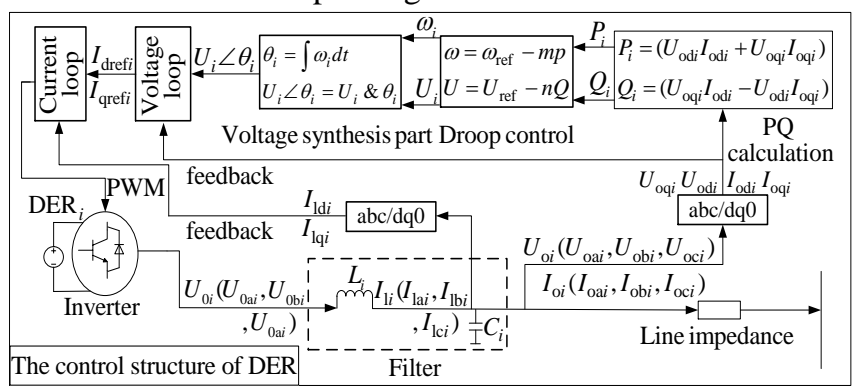

Fig. 4 Simplified inverter control structure of the $i$-th DER

1) The control system in Fig. 1 is discretized with the sampling time period chosen as T. The output voltage and frequency of droop control at $\mathrm{t}=K \mathrm{~T}$ are denoted by $U_{i}(K)$ and $\omega_{i}(K)$ respectively.

2) $U_{i}(K)$ and $\omega_{i}(K)$ will be transferred to the voltage synthesis part. This process can be represented as

$$
U_{i} \angle \theta_{i}(K)=f_{1}\left(U_{i}(K), \omega_{i}(K)\right)
$$

3) Park Transformation is applied to $U_{i} \angle \theta_{i}(K)$, with the results denoted by $U_{\text {drefi }}(K)$ and $U_{\text {qrefi }}(K)$. According to the structure of voltage and current double closed loop in [27], $U_{\text {drefi }}(K)$ and $U_{\text {qrefi }}(K)$ are also the inputs of voltage loop. After inputting $U_{\text {drefi }}(K)$ and $U_{\text {qrefi }}(K)$ to the voltage loop, $I_{\text {drefi }}(K)$ and $I_{\text {qrefi }}(K)$ can be obtained. Meanwhile, the two variables are the inputs of current loop. The process can be summarized as

$$
\left\{\begin{array}{l}
U_{\text {drefi }}(K)=f_{2}\left(U_{i} \angle \theta_{i}(K)\right) \\
U_{\text {qrefi }}(K)=f_{3}\left(U_{i} \angle \theta_{i}(K)\right) \\
I_{\text {drefi }}(K)=f_{4}\left(U_{\text {drefi }}(K), U_{\text {odi }}(K), U_{\text {oqi }}(K), I_{\text {odi }}(K)\right) \\
I_{\text {qrefi }}(K)=f_{5}\left(U_{\text {qrefi }}(K), U_{\text {oqi }}(K), U_{\text {odi }}(K), I_{\text {oqi }}(K)\right)
\end{array}\right.
$$

4) $I_{d r e f i}$ and $I_{\text {qrefi }}$ will be transferred to the current loop. Meanwhile, the PWM trigger signal will be obtained by current loop at first. Then, the trigger signal will be used to generate the PWM signal. After that, the PWM signal will be used to control the inverter. The signal $U_{0 i}(k+1)$, including components $U_{0 a i}(k+1), U_{0 b i}(k+1)$ and $U_{0 c i}(k+1)$, will also be obtained. Finally, through the LC filter, $U_{o i}(K+1)$ (including $U_{o a i}(k+1), U_{o b i}(k+1)$ and $\left.U_{o c i}(k+1)\right)$ and $I_{o i}(K+1)$ (including $U_{0 a i}(k+1), U_{0 b i}(k+1)$, and $\left.U_{0 c i}(k+1)\right)$ will be obtained. The process can be expressed as

$$
\left\{\begin{array}{l}
P W M_{i}(K)=f_{6}\left(I_{\text {drefi }}(K), I_{\text {qrefi }}(K), I_{\text {ldi }}(K), I_{\text {lqi }}(K)\right) \\
U_{0 i}(K+1)=f_{7}\left(P W M_{i}(K), U_{D C i}, Z_{\text {Inverteri }}\right) \\
U_{o i}(K+1)=f_{8}\left(U_{0 i}(K+1), L_{i}, C_{i}, Z_{\text {Linei }}\right) \\
I_{o i}(K+1)=f_{9}\left(U_{0 i}(K+1), L_{i}, C_{i}, Z_{\text {Linei }}\right)
\end{array}\right.
$$

5) The signals of $U_{o d i}(K+1), U_{o q i}(K+1), I_{o d i}(K+1)$ and $I_{\text {oqi }}(K+1)$ will be obtained by the Park transformation on $U_{o i}(K+1)$ and $I_{o i}(K+1)$. Then these four signals will be transferred to the PQ calculation part. Meanwhile, $P_{i}(K+1)$ and $Q_{i}(K+1)$ can be obtained. The process can be written as 
$\left\{\begin{array}{l}P_{i}(K+1)=f_{10}\left(U_{o d i}(K+1), U_{o q i}(K+1), I_{o d i}(K+1), I_{o q i}(K+1)\right) \\ Q_{i}(K+1)=f_{11}\left(U_{o d i}(K+1), U_{o q i}(K+1), I_{o d i}(K+1), I_{o q i}(K+1)\right)\end{array}\right.$

6) $P_{i}(K+1)$ and $Q_{i}(K+1)$ will be transferred to the droop control part, and $U_{i}(K+1)$ and $\omega_{i}(K+1)$ can also be obtained in this part. The process can be expressed as

$$
\left\{\begin{array}{l}
U_{i}(K+1)=f_{12}\left(P_{i}(K+1)\right) \\
\omega_{i}(K+1)=f_{13}\left(Q_{i}(K+1)\right)
\end{array}\right.
$$

In summary, the output of droop control part at $t=(K+1) \mathrm{T}$ can be obtained from the output data of droop control at $t=K T$. The initial parameters for MPC include $Z_{\text {Inverteri }}, Z_{\text {Linei }}, L_{i}, C_{i}$ and $U_{D C i}$. Since the running time in the MG is continuous, a D-A converter is needed at the output of MPC.

Based on the above analysis, the power output data at $\mathrm{t}=(\mathrm{K}+1) T$ can be predicted from the relevant data at $\mathrm{t}=\mathrm{K} T$.

\section{2 Data pre-processing by EMD}

It is often difficult to predict WT and PV generation because of strong random volatility. Therefore, to achieve a better prediction result, EMD is used to decompose the volatile historical data into finite intrinsic modal functions (IMFs) and a remainder. Local characteristics in different time periods of the original data are contained in IMFs. If the predictive values of each IMF and remainder can be obtained by using multiple ELMs, the predictive value of generation power will be obtained by superimposing the first two predictive values. An IMF must satisfy two conditions: 1) The difference between the number of extremums and the number of zeros is either 0 or $\pm 1 ; 2$ ) The mean value of the upper envelope line and the lower envelope line at any time must be zero. The decomposition process is as follows: All the maximum points of the original data $x(t)$ are found to form the upper envelope line. All the minimum points of $x(t)$ are found to form the lower envelope line. The mean values of upper and lower envelope lines are denoted as $m_{l}$. A new variable $h(t)$ can be obtained by using $x(t)$ minus $m_{l}$. If $h(t)$ is not an IMF, the decomposition will continue; otherwise, the above decomposition is carried out for the obtained remainder until the maximum iteration number is reached or the remainder becomes a monotone function [30]. The detailed process is illustrated in Fig. 5.

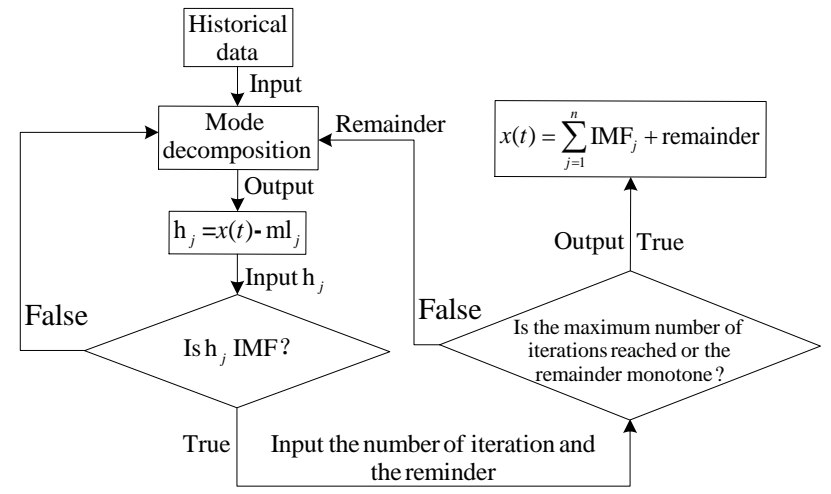

Fig. 5 The process of EMD

In summary, EMD can decompose a set of data into multiple IMFs and one remainder in time domain. To better explain the above decomposition process, a group of PV power generation in a rainy day in a certain area of China is taken as an example, and we add the simulation results as below: Empirical Mode Decomposition
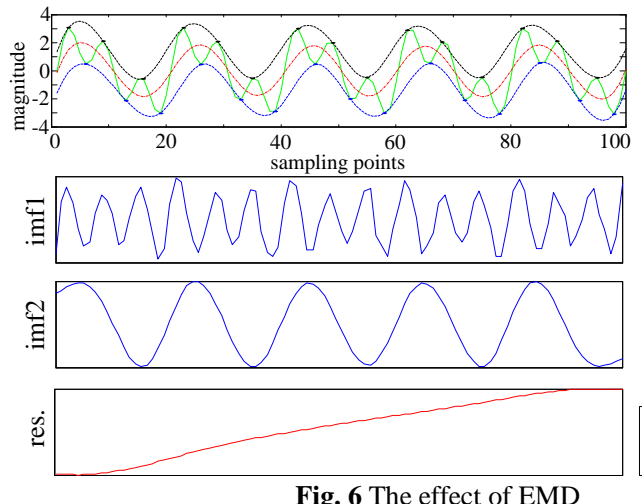

original signal
upper envelope
lower envelope mean envelop

Fig. 6 The effect of EMD

In the above figure, we can see that PV power generation in rainy season is more regular. However, due to the irregularity of the generation curve, the prediction is not easy. Moreover, the upper and lower envelopes in EMD decomposition are the lines between the maximum and the minimum of the data curve as shown in Fig. 6. After two decompositions with IMF1 and IMF2, the resulting remainder is monotonous, so the EMD stops. From Fig. 6, we can see that the volatility of IMF1 is still high, so it is suitable for prediction with ELM; the regularity of IMF2 and remainder is strong, so it is suitable to use MPC to complete the prediction. Therefore, we can find that EMD can better predict the future trend of data. III. 3 Application of ELM

ELM is a fast training method for single hidden layer feed-forward neural networks [31]. The initial parameters for ELM include historical data, operation time, number of neurons and initial weight, etc. This method is briefed below.

A set of training samples is chosen as $\mathrm{T}_{\mathrm{ELM}}=\left\{\left(\mathrm{x}_{\mathrm{i}}, \mathrm{y}_{\mathrm{i}}\right), \mathrm{i}=1, \cdots, \mathrm{L}\right\}$, where $x_{i} \in R^{n}, y_{i} \in R^{n}$. The ELM model with $N$ hidden layer nodes can be expressed as

$$
\sum_{i=1}^{N} \beta_{i} g\left(\alpha_{i} x_{i}+\gamma_{i}\right)=\boldsymbol{H} \boldsymbol{\beta}
$$

According to (6), the weights of the hidden layer to the output layer can be obtained by the least square solution of the following problem:

$$
\min _{\boldsymbol{\beta}}\|\boldsymbol{H} \boldsymbol{\beta}-\boldsymbol{Y}\|
$$

where \|\| is Frobenius norm of matrix. The solution of (7) is $\hat{\boldsymbol{\beta}}=\boldsymbol{H}^{+} \boldsymbol{Y}, \boldsymbol{H}^{+}$is the Moore-Penrose generalized inverse matrix of $\boldsymbol{H}$. According to the method of ELM, the mathematical model of the MG can be built quickly from histori- 
cal data. The targeted output of ELM is the active power of each DER. For this purpose, the activation function is selected as sigmoid function; the number of neurons in hidden layer is chosen as 20 for each ELM; the weights of input matrix are given randomly with an interval of $[0,1]$ the beginning of the training; and the regularization parameter is selected as 1e5. When the weight of the input layer to the hidden layer is determined, the weight of the hidden layer to the output layer can be obtained according to (6) and (7), thus the training of ELM is completed.

\section{4 Output the predicted data}

The procedure of the PCM can be divided as below:

1. Firstly, EMD is used to preprocess the historical data. After preprocessing, the historical data is decomposed into multiple intrinsic mode functions (IMFs) and one remainder.

2. For this series of IMFs, the first $s$ items are selected for multi-ELMs prediction (the first $s$ term is still fluctuating, so it is not suitable for MPC). Other items (including the remainder) are predicted by MPC.

3. The predicted data of historical data are obtained by superposing the predicted data of IMFs and remainder.

Moreover, for $m$ ELMs, there is a weight processing procedure as below.

1) Weight processing for ELMs

There are $m$ ELMs used to predict, and the weight processing is carried out according to the predictive accuracy of each ELM. To make the predictive model with smaller predictive errors having a larger weight, the following method is used to design the weight of each ELM.

$$
\begin{aligned}
& e_{i}(K)=y(K)-y_{\mathrm{ELM}_{i}}(K) \\
& c_{\mathrm{ELM}_{i}}(K)=\left[1-\left|e_{i}(K)\right| / \sum_{i=1}^{m}\left|e_{i}(K)\right|\right] /(m-1)
\end{aligned}
$$

The output data of ELMs after weight processing are given below:

$$
y_{\mathrm{ELM}}(K)=\sum_{i=1}^{m} c_{i}(K) y_{\mathrm{ELM}_{i}}(K)
$$

2) Obtain the predicted data

The final predicted data at $t=K T$ is $\hat{y}(K)=y_{\mathrm{ELM}}(K)+y_{\mathrm{MPC}}(K) \quad, \quad(y=U / y=\omega)$.

3) Retrain ELMs

If there is a large error between the predicted data and the actual historical data, the ELMs will be retrained and returned to step 1) until the error requirement is met.

The specific prediction process is also show in Fig. 7. According to this figure, the parameter $s$ and $m$ will affect the prediction accuracy. Therefore, a parameter selection method is proposed in this paper to select these parameters. The specific design is as the follows.

\section{Step 1. Design of the objective function}

For PCM, smaller prediction error is targeted, and the mean average percentage error (MAPE) is selected as the objective function to be minimized. The corresponding equation is

$$
\min \text { MAPE }=\frac{1}{N} \sum_{i=1}^{N} \frac{\left|x_{\text {train. } i}-x_{\text {train. } i}^{\prime}\right|}{x_{\text {train. } . i}^{\prime}} \times 100 \%
$$

Step 2. Using any existing intelligent algorithms, such as genetic algorithm or the improved PSO algorithm proposed in this paper, to obtain the solution.

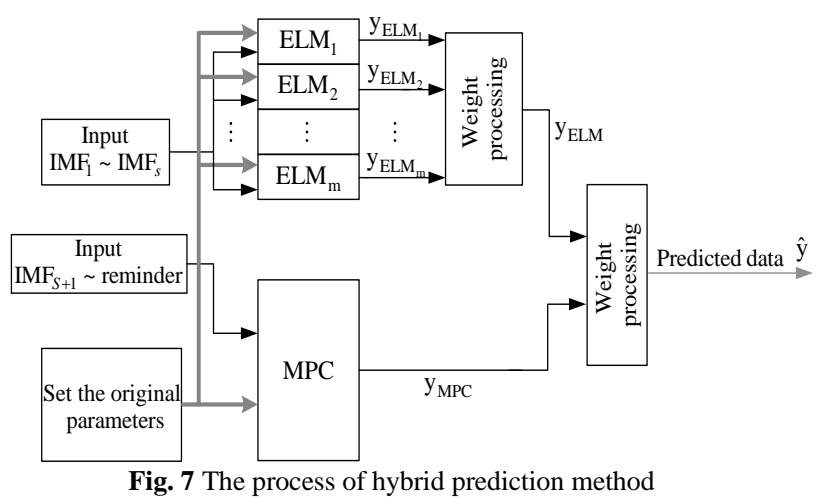

IV. The solution of interruption in data transmission

In the process of optimization, the predicted data are transmitted to the EMS part through the sensor. If the communication is interrupted, the entire energy management process will be affected. Therefore, based on the vulnerability assessment and prospect theory, a neighbor prediction method is proposed to solve the problem.

\section{1 Vulnerability assessment}

The physical devices such as DER can be classified as the physical nodes; and communication devices such as sensors can be classified as the cyber nodes. All nodes have certain vulnerabilities. Furthermore, since each DER corresponds to a sensor, the vulnerability of physical nodes and cyber nodes is related to each other [32]. Each DER and its corresponding communicator constitute a cyber-physical node (CPN). For a CPN, the cyber and physical vulnerabilities should be combined. If the $i$-th physical node cannot transmit its predicted data to EMS, then the neighbor CPN with the lowest vulnerability can be selected, which is denoted as the $j$-th node. Different to the $i$-th physical node, the $j$-th physical node makes use of historical data of the $i$-th node to predict and transmit the predicted data to EMS. Moreover, using the vulnerability prospective value will be more favorable for future path decision. The evaluation method on the $j$-th CPN is as follows.

1) Select the vulnerability index of physical nodes. For a physical node, the vulnerability of a node is the extent of the impact on the system when the node is removed from the system. In this paper, the source-flow path partition method is used as the assessment method, which is a simple and effective method to analyze the vulnerability. By using this method, the set of all power paths can be obtained as $L_{q}, q=1 \sim N_{L}$, where $N_{L}$ is the total number of electrical paths. The vulnerability index of the $l$-th branch of the path Lq is defined as $T_{L q(l)}$, which satisfies the following equation.

$$
T_{L q(l)}=d_{L q(l)} I_{1, L q(l)} I_{2, L q(l)} \lambda_{L q(l)}
$$

Based on (11), the cumulative vulnerability of the $l$-th branch in all possible electrical paths can be defined as the vulnerability of the branch. Meanwhile, the maximum vulnerability of the branch connected to the $j$-th node can be defined as the vulnerability of node $j$, that is,

$$
\begin{gathered}
C_{p}[\operatorname{branch}(l)]=\sum_{q=1}^{N_{L}} T_{L q(l)} \\
C_{p}[N(j)]=\max _{l}\left\{C_{p}[\operatorname{branch}(l)]\right\}
\end{gathered}
$$


where branch $l$ includes the $j$-th node ; $C_{p}[\operatorname{branch}(l)]$ is the vulnerability of the $l$-th branch; and $C_{p}[N(j)]$ is the vulnerability of the $j$-th node.

2) Select the vulnerability index on cyber node. The main purpose here is to evaluate the vulnerability of communicators. The index at a node includes three components: the importance degree of this node, the number of failures of this node during the year, and the protective measures (e.g., firewall) of this node. The specific values can be obtained by the expert scoring method, and the specific equation is

$$
C_{C}[N(j)]=C_{C 1 j}+C_{C 2 j}+C_{C 3 j}
$$

The specific scores are obtained by experts according to the corresponding scoring criteria as Table I.

\begin{tabular}{|c|c|c|c|}
\hline $\begin{array}{c}\text { Importance } \\
\text { degree }\end{array}$ & $\begin{array}{l}\text { The number } \\
\text { of failures }\end{array}$ & $\begin{array}{c}\text { Protective } \\
\text { measures }\end{array}$ & Score \\
\hline $\begin{array}{l}\text { Node is not important. } \\
\text { It doesn't damage the } \\
\text { cyber layer after it's bro- } \\
\text { ken. }\end{array}$ & $\begin{array}{l}\text { Basically no } \\
\text { failures }\end{array}$ & $\begin{array}{l}\text { Protective } \\
\text { measures are } \\
\text { sufficient }\end{array}$ & 1 \\
\hline $\begin{array}{l}\text { Node isn't too im- } \\
\text { portant. Node damage can } \\
\text { cause a lower level of } \\
\text { damage. }\end{array}$ & $\begin{array}{l}\text { The number of } \\
\text { failures during the } \\
\text { past year is less } \\
\text { than } 5\end{array}$ & $\begin{array}{l}\text { Protective } \\
\text { measures are } \\
\text { basically } \\
\text { perfect }\end{array}$ & 3 \\
\hline $\begin{array}{l}\text { Node is important. } \\
\text { Node damage can cause } \\
\text { moderate damage }\end{array}$ & $\begin{array}{l}\text { The number of } \\
\text { failures during the } \\
\text { past year is be- } \\
\text { tween } 5 \text { and } 10\end{array}$ & $\begin{array}{l}\text { Protective } \\
\text { measures are } \\
\text { general }\end{array}$ & 5 \\
\hline $\begin{array}{l}\text { Node is important. } \\
\text { Node damage can cause } \\
\text { serious damage }\end{array}$ & $\begin{array}{l}\text { The number of } \\
\text { failures during the } \\
\text { past year is be- } \\
\text { tween } 10 \text { and } 20\end{array}$ & $\begin{array}{l}\text { Protective } \\
\text { measures are } \\
\text { poor }\end{array}$ & 7 \\
\hline $\begin{array}{l}\text { Node is very important. } \\
\text { Node damage can cause } \\
\text { very serious damage }\end{array}$ & $\begin{array}{l}\text { The number of } \\
\text { failures during the } \\
\text { past year is more } \\
\text { than } 20\end{array}$ & $\begin{array}{l}\text { Basically } \\
\text { no protec- } \\
\text { tive } \\
\text { measures. }\end{array}$ & 9 \\
\hline
\end{tabular}

3) Vulnerability indexes are pretreated as follows. The max-min fairness method is adopted for this pretreatment due to its simplicity to obtain a dimensionless quantity. That is, for any given $j$-th $\mathrm{CPN}$, the calculation for the i-th index is done by: $\xi_{i j}=\left(C_{c i j}-C_{i \min }\right) /\left(C_{c i \max }-C_{c i \min }\right)$.

4) Now, entropy weighting method [33] is used to assign the weight of indexes. This method can assign higher weights to important indexes. After weighting, the weights of vulnerability indexes are given in Table II.

\begin{tabular}{c|cccc}
\multicolumn{7}{c}{ Table II Weights of vulnerability indexes } \\
\hline $\begin{array}{l}\text { Vulnera- } \\
\text { bility } \\
\text { indexes }\end{array}$ & $\begin{array}{l}\text { Physical } \\
\text { vulnerability } \\
\text { index }\left(\xi_{1 j}\right)\end{array}$ & $\begin{array}{l}\text { Cyber node } \\
\text { importance } \\
\left(\xi_{2 j}\right)\end{array}$ & $\begin{array}{l}\text { The number } \\
\text { of failure } \\
\left(\xi_{3 j}\right)\end{array}$ & $\begin{array}{l}\text { Protective } \\
\text { measures } \\
\left(\xi_{4 j}\right)\end{array}$ \\
\hline \multicolumn{1}{c|}{ Weight } & $\psi_{1}$ & $\psi_{2}$ & $\psi_{3}$ & $\psi_{4}$
\end{tabular}

5) By applying the cumulative prospect theory, the vulnerability prospect value of each CPN is obtained. Cumulative prospect theory is a decision theory, which can consider the uncertainty of the objective and subjective preference of the decision-maker. In this theory, the core part is the prospect value, which is determined by the value function $v\left(\xi_{i j}\right)$ and the weight function $\pi\left(\psi_{i}\right)$. The equation of cumulative prospect value is given as below:

$$
V_{j}=\sum_{i=1}^{n} \pi_{i}^{+} v\left(\xi_{i j}\right)+\sum_{i=1}^{n} \pi_{i}^{-} v\left(\xi_{i j}\right)
$$

The corresponding equations are as follows:

$$
\begin{cases}v\left(\xi_{i j}\right)=\xi_{i j}^{\alpha}, & \Delta \xi_{i j} \geq 0 \\ v\left(\xi_{i j}\right)=-\lambda(-\xi)_{i j}^{\beta}, & \Delta \xi_{i j}<0\end{cases}
$$

$$
\pi\left(\psi_{i}\right)=\left\{\begin{array}{l}
\pi_{i}^{+}=\frac{\psi_{i}}{\left(\psi_{i}^{\gamma^{+}}+\left(1-\psi_{i}\right)^{\gamma^{+}}\right)^{1 / \gamma^{+}}}, \quad \Delta \xi_{i j} \geq 0 \\
\pi_{i}^{-}=\frac{\psi_{i}^{\gamma^{-}}}{\left(\psi_{i}^{\gamma^{-}}+\left(1-\psi_{i}\right)^{\gamma^{-}}\right)^{1 / \gamma^{-}}}, \quad \Delta \xi_{i j}<0
\end{array}\right.
$$

6) Based on 1) 5), the prospective vulnerability of each CPN can be obtained. A smaller prospective value indicates a smaller vulnerability of the CPN.

\section{2 Neighbor-prediction method}

Based on the selected CPN, the neighbor-prediction process can be completed. Taking the data transmission of the $i$-th DER as an example, the corresponding steps of neighbor- prediction process are as follows. Firstly, based on the results of the vulnerability assessment, the DER with the least vulnerability in the neighbor DERs of the $i$-th DER is selected. Then in the selected DER, the power generation data of the $i$-th DER are predicted. Finally, the predicted data will be transmitted to the EMS by the sensor of this neighbor DER. Based on the above process, the normal operation of EMS can be ensured even if there is interruption. It is also found that when the communication of the predicted data of a certain device is interrupted, its neighboring devices will be used to carry out the related prediction. The above process can be understood as that the predicted data by neighboring equipment will compensate the lost data when required. Therefore, the contributed method is also called as "predictive compensation". V .The optimization models

Since the generation of renewable energy is uncertain, and a large number of interruptible loads and flexible loads exist in MGs, DSR has been widely applied to the EMS of MG. The use of DSR means that we can properly take advantage of flexible and interruptible loads and energy storage facilities so as to shift or reduce unnecessary peaks, thus maintain reliable and economic operations of the MG. In this paper, the relevant studies of the DSR are as follows.

\section{V.1 The objection function}

The operation cost function of controllable DER is

$$
C_{\mathrm{DER} g}(t)=a P_{\mathrm{DER} g}^{2}(t)+b P_{\mathrm{DER} g}(t)+c \delta_{\mathrm{DER} g}(t)+d
$$

When there is power exchange between MG and the main grid, the power exchange cost of MG is

$$
\begin{gathered}
C_{\text {grid }}(t)=c_{\text {grid }}(t) P_{\text {grid }}(t) \\
c_{\text {grid }}(t)= \begin{cases}c_{\text {buy }}, & P_{\text {grid }} \geq 0 \\
c_{\text {sell }}, & P_{\text {grid }}<0\end{cases}
\end{gathered}
$$

A positive value of $P_{\text {grid }}(t)$ means MG buys electricity from grid, while a negative $P_{\text {grid }}(t)$ means MG sells electricity to the grid.

The cost of energy storage battery is 


$$
C_{\text {bat }}(t)=\lambda_{\text {bat }} P_{\text {bat }}^{2}(t)
$$

The cost of the MG to compensate owners of interruptible load [34] is

$$
C_{I l i}(t)=\varepsilon_{I l i}(t) \rho_{I l i}(t) P_{I l i}(t)
$$

Based on [35], the cost of the MG to compensate owners of flexible load is

$$
C_{f i j}(t)=\varepsilon_{f j}(t) \rho_{f i j}(t) P_{f f j}(t)
$$

Under the assumption of voltage stability, the objective function aiming at the minimum total operating cost of MG can be formulated as follows

$$
\begin{aligned}
& \min F=\sum_{t=1}^{S} \sum_{g=1}^{N} C_{\mathrm{DERg}}(t)+\sum_{t=1}^{S} C_{\text {grid }}(t)+ \\
& \sum_{t=1}^{S} C_{\mathrm{bat}}(t)+\sum_{t=1}^{S} \sum_{i=1}^{I} C_{I l i}(t)+\sum_{t=1}^{S} \sum_{j=1}^{J} C_{f f j}(t)
\end{aligned}
$$

\section{2 System constraints}

The constraints of DERs are formulated below:

1) The constraint on active power is

$$
P_{\mathrm{DER} g}^{\min } \delta_{\mathrm{DER} g}(t) \leq P_{\mathrm{DER} g}(t) \leq P_{\mathrm{DER} g}^{\max } \delta_{\mathrm{DER} g}(t)
$$

2) The ramp rate constraint is:

$$
\left|P_{\mathrm{DER} g}(t)-P_{\mathrm{DERg}}(t-1)\right| \leq \Delta P_{\mathrm{DER} g}
$$

3) The constraint on minimum running time is given as

$$
\begin{aligned}
& \delta_{\mathrm{DER} g}(t)-\delta_{\mathrm{DER} g}(t-1) \leq \delta_{\mathrm{DERg}}\left(\tau_{1}\right), \\
& \forall \tau_{1} \in\left[t+1, \min \left(t+T^{u p}-1, T^{\text {opt }}\right)\right]
\end{aligned}
$$

4) The constraint on minimum downtime

$$
\begin{gathered}
\delta_{\mathrm{DER} g}(t-1)-\delta_{\mathrm{DERg}}(t) \leq 1-\delta_{\mathrm{DERg}}\left(\tau_{2}\right), \\
\forall \tau_{2} \in\left[t+1, \min \left(t+T^{\text {down }}-1, T^{\text {opt }}\right)\right]
\end{gathered}
$$

The MG system satisfies the following constraints.

1) The constraint on power balance is given as:

$$
\begin{aligned}
& \sum_{g=1}^{N+R} P_{\mathrm{DERg}}(t)+P_{g r i d}(t)=\sum_{i=1}^{I} P_{c l i}(t)\left[1-\rho_{c l i}(t)\right] \\
& +\sum_{j=1}^{J} \delta_{f f j}(t) P_{f j}(t)+\sum_{k=1}^{\Omega} P_{n l k}(t)+\eta P_{b a t}(t)
\end{aligned}
$$

2) The energy storage battery satisfies:

$$
P_{\text {bat }}^{\min } \leq P_{\text {bat }}(t) \leq P_{\text {bat }}^{\max }
$$

3) The amount of power exchanged between MG and the main grid satisfies:

$$
P_{\text {grid }}^{\min } \leq P_{\text {grid }}(t) \leq P_{\text {grid }}^{\max }
$$

4) The constraint on interruptible load is:

$$
\rho_{I l i}^{\min } \leq \rho_{c l i}(t) \leq \rho_{I l i}^{\max }
$$

5) The flexible load needs to satisfy the following constraint:

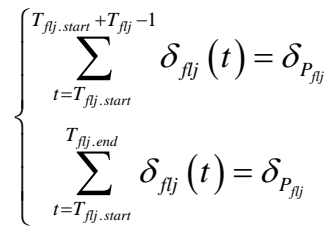

The meaning of (33) is that the flexible load only runs within a given time period and cannot stop once it is running before the task is completed.

\section{V.3 An improved PSO algorithm}

In the optimization problem considered in this paper, the objective function in (24) includes quadratic items, such as (18) and (21), and also discontinuous items such as (19). Therefore, it is not a convex optimization problem, and a new PSO algorithm is designed to solve this mixed integer nonlinear optimization problem. . Actually, PSO is an evolutionary computing algorithm. To improve the convergence speed to global optimum, a particle aggregation degree based-PSO algorithm is proposed here by taking advantage of genetic algorithms. Details are given below.

In the beginning of optimization, the method of roulette is used to select the initial particle, and then crossover and mutation are taken to expand the searching range of the algorithm (i.e., prevent it being trapped into a local optimal solution).The convergence speed is improved by the method of elite selection [37]. The flow chart of the algorithm is shown in Fig. 8.
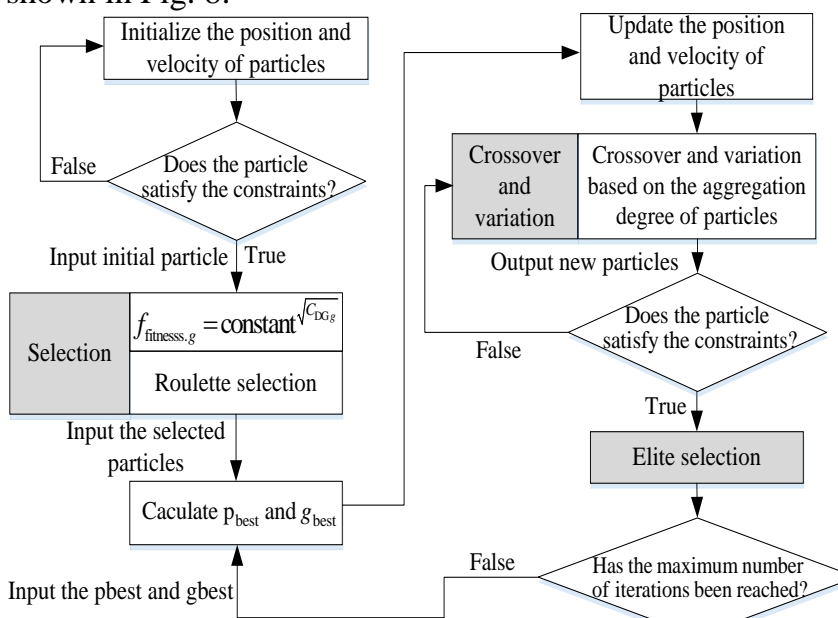

Output new particles $\mathbf{Y}$ Does the particle False satisfy the constraints?

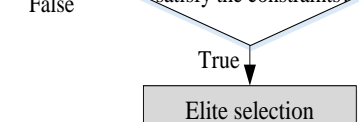
Elite selection

Detailed steps of the improved PSO are as follows:

Step 1. The initial particle (each particle represents a dispatch strategy) is selected and the position and velocity of each particle are assigned with initial values.

Step 2. Select particle population and set the fitness function as $f_{\text {fitnesss }}=$ constant ${ }^{\sqrt{|F|}}$, where $0.5<$ constant $<1 ; F$ is the total operating cost of MG. According to the fitness function value of each particle, roulette selection is carried out [37]. This scheme can expand the searching range of PSO.

Step 3. Calculate individual optimal value $\mathrm{p}_{\text {best }}$ and the overall optimal value $g_{\text {best }}$. The position and velocity of particles are updated according to the standard PSO iterative formula [38].

Step 4. Crossover and mutation are carried out according to the aggregation density of particles to ensure that PSO is not trapped into local optima. By studying the variation of fitness function values of all particles in the population, the quantitative description of the aggregation degree of each particle can be obtained. To facilitate discussion, assume that the total number of particles in the particle swarm is $n$; the fitness function of the $i$-th particle is denoted by $f_{\text {fitness. } i}$; and the average fitness function of each particle is $f_{\text {fitness.avr.n }}$. Then the aggregation degree of the group can be expressed as $\rho=\sum_{i=1}^{n}\left[\left(f_{\text {fitness. } i}-f_{\text {fitness.avr.n }}\right) / f_{\rho}\right]^{2}$, where $f_{\rho}$ is used to limit the size of $\rho$, and its values can be obtained as: $f_{\rho}=\max \left\{1, \max _{i \in n}\left|f_{\text {fitness. } i}-f_{\text {fitness.avr. }}\right|\right\}$. 
The crossover and variation rates are then decided. crossover rate :

$$
\begin{aligned}
& P_{C R}^{k}=\left(p_{C R}^{\max }-p_{C R}^{\min }\right)\left(\rho_{k} / n\right)^{2}+\left(p_{C R}^{\min }-p_{C R}^{\max }\right)\left(2 \rho_{k} / n\right)+p_{C R}^{\max } \\
& \text { mutation rate : } \\
& P_{M R}^{k}=\left(p_{M R}^{\max }-p_{M R}^{\min }\right)\left(\rho_{k} / n\right)^{2}+\left(p_{M R}^{\min }-p_{M R}^{\max }\right)\left(2 \rho_{k} / n\right)+p_{M R}^{\max }
\end{aligned}
$$

Step 5. The next generation particle population is selected by the elite selection. Thus, the current individual with the best fitness function can be directly selected into the next generation population.

Step 6. If the allowed maximum number of iterations is achieved, then jump out of the iteration; otherwise, continue to iterate.

Convergence of the improved PSO has been verified by the case studies in Section VI, and in particular results from Fig. 16 and 17 show that the improved PSO algorithm has significantly reduced the total cost (comment: here you can also add something on convergence speed, i.e. computing time when it converges to the optimal solution). This new algorithm also improves the reliability of the ordinary PSO. In fact, we have designed the crossover rate and the mutation rate according to the aggregation degree of these particles. In this way, the particles with higher aggregation degree will have higher crossover rate and mutation rate. Therefore, it will help the algorithm to jump out of the local optimum and improve the accuracy of the optimization solution. This is also verified by Fig. 16 and 17 that the new PSO algorithm can reduce the inaccurate minimal cost of $\$ 522.6$ from ordinal PSO to a much smaller value of $\$ 293.5$.

\section{Simulation and analysis}

Simulations are performed in this section to verify the microgrid energy management methodologies proposed in the previous three sections.

The MG structure in Fig. 1 is simulated and verified. The system consists of four kinds of DERs, one energy storage battery and three kinds of loads connected to the main grid. The related information of each DER is shown in Table III. The parameters of controllable DERs are presented in Table IV. The parameters of the energy storage are provided in Table V. A time-of-use tariff with peak, standard and off-peak prices for the MG to purchase power from or sell power to the grid is given in Table VI. The operation data of flexible load can be found in Table VII. The corresponding parameters are as Table VIII. The power exchange limit between MG and main grid is $10 \mathrm{~kW}$.

Table III Data of microgrid distributed installed power generation capacity

\begin{tabular}{ccc}
\hline $\begin{array}{c}\text { The type of } \\
\text { DER }\end{array}$ & $\begin{array}{c}\text { Minimum output } \\
\text { power/kW }\end{array}$ & $\begin{array}{c}\text { Maximum output } \\
\text { power/kW }\end{array}$ \\
\hline MT & 3 & 30 \\
FC & 3 & 30 \\
PV & 0 & 85 \\
WT & 0 & 50 \\
\hline
\end{tabular}

\begin{tabular}{|c|c|c|c|c|c|}
\hline \multicolumn{6}{|c|}{ Purchase price $/ \$ / \mathrm{kWh} \quad$ Selling price $/ \$ / \mathrm{kWh}$} \\
\hline \multicolumn{2}{|c|}{ Peak } & \multicolumn{2}{|r|}{0.18} & \multicolumn{2}{|r|}{0.16} \\
\hline \multicolumn{2}{|c|}{ Standard } & \multicolumn{2}{|r|}{0.10} & \multicolumn{2}{|r|}{0.08} \\
\hline \multicolumn{2}{|c|}{ Off-peak } & \multicolumn{2}{|r|}{0.06} & \multicolumn{2}{|r|}{0.04} \\
\hline \multicolumn{6}{|c|}{ Table VII Relevant parameters of flexible load } \\
\hline $\begin{array}{c}\text { Flexible } \\
\text { loads }\end{array}$ & $\begin{array}{l}\text { Pow- } \\
\text { er/kW }\end{array}$ & $\begin{array}{c}\text { Earliest } \\
\text { start time } / \mathrm{h}\end{array}$ & $\begin{array}{l}\text { Latest end } \\
\text { time/h }\end{array}$ & $\begin{array}{c}\text { Running } \\
\text { time/h }\end{array}$ & $\begin{array}{c}\text { Compensation } \\
\text { cost } / \$ / \mathrm{kWh}\end{array}$ \\
\hline 1 & 13 & 9 & 19 & 2.5 & 0.08 \\
\hline 2 & 15 & 13 & 22 & 0.5 & 0.08 \\
\hline 3 & 7.3 & 5 & 17 & 1 & 0.07 \\
\hline 4 & 12.4 & 10 & 22 & 0.5 & 0.06 \\
\hline 5 & 8.08 & 6 & 24 & 3 & 0.06 \\
\hline \multicolumn{6}{|c|}{ Table VIII Relevant parameters of interruptable load } \\
\hline $\begin{array}{l}\text { Interruptible } \\
\text { load }\end{array}$ & $\begin{array}{l}\text { Pow- } \\
\text { er/kW }\end{array}$ & $\begin{array}{c}\text { Earliest } \\
\text { start time } \\
\text { /h } \\
\end{array}$ & $\begin{array}{c}\text { Latest } \\
\text { end } \\
\text { time/h }\end{array}$ & $\begin{array}{c}\text { Running } \\
\text { time/h }\end{array}$ & $\begin{array}{l}\text { Compensa- } \\
\text { tion } \\
\text { cost } \$ \$ \mathrm{kWh}\end{array}$ \\
\hline 1 & $0 \sim 14$ & 0 & 11 & 0.8 & 0.16 \\
\hline 2 & $0 \sim 10$ & 12 & 18 & 0.6 & 0.16 \\
\hline 3 & $0 \sim 8$ & 19 & 24 & 0.5 & 0.14 \\
\hline
\end{tabular}

\begin{tabular}{cccccc}
\hline \multicolumn{5}{c}{ Table IV Relevant parameters of controllable DERs } \\
\hline Type & $\mathrm{a}$ & $\mathrm{b}$ & $\mathrm{c}$ & $\mathrm{T}_{\text {up }} / \mathrm{h}$ & $\mathrm{T}_{\text {down }} / \mathrm{h}$ \\
\hline MT & 0.00624 & 0.146 & 0 & 1 & 1 \\
$\mathrm{FC}$ & 0.00498 & 0.183 & 0 & 1 & 1 \\
\hline \multicolumn{5}{c}{ Table V Parameters of battery } \\
\hline \multicolumn{3}{c}{ Parameter } & Value & \multicolumn{3}{c}{ Parameter } & Value \\
\hline \multicolumn{3}{c}{$\begin{array}{c}\text { Power rating } \\
\text { / } \mathrm{kW}\end{array}$} & 30 & Rated capacity & 30 \\
cost coefficient & 0.03 & Range of capacity & $0.1 \sim 0.9$ \\
\hline
\end{tabular}

Table VI The purchasing and selling prices of electricity in peak,
VI. 1 Verification of the hybrid prediction method

In Fig. 1, the PV generation is taken as the example to verify the effect of hybrid prediction method proposed in this paper. To get closer to the actual situation, the generated power in sunny and rainy days are predicted respectively. In addition to these two weather conditions, we also verify the prediction effect of the proposed method under uncertain weather conditions.. The relative error, MAPE and root mean square error (RMSE) are used to illustrate the prediction effect. The corresponding formulas can be found from [40].

The effectiveness of hybrid prediction method is compared with the method using ELMs $(\mathrm{m}=5)$ or MPC alone. As shown in Fig. 9 (a) and (c), the horizontal and vertical axes represent time and predicted power data, respectively. It is found that there is no significant difference between the three prediction methods in sunny days. In rainy days, the system fluctuates greatly and the adaptability of the MPC method is poor, which leads to the biggest error between the MPC prediction data and the real data. The effect of using ELM to predict is better than that of MPC. However ELM is a random method in selecting input weights, and different ELM models have different adaptive ability. Therefore, the prediction error of ELM model is uncertain. By using the newly proposed hybrid method, the predicted data are the closest to the real data.

From Fig. 9. (a) and (c), the PV system usually generates power from 6: 00 to 20: 00 . To study the prediction effect more intuitively, the prediction error in this time period is expressed as shown in Fig. 9. (b), (d) and Table IX. It can be seen that when the weather is sunny, the prediction results of the three forecasting methods are relatively accurate. When the weather is rainy, the actual generation power fluctuates greatly. In a rainy day, the prediction relative error obtained by using MPC or ELM alone will suddenly increase, which will directly affect the prediction effect. The relative error obtained by combining the two methods in this paper is the smallest. This shows that the method proposed in this paper is more applicable to different weather conditions with greater randomness and volatility. 


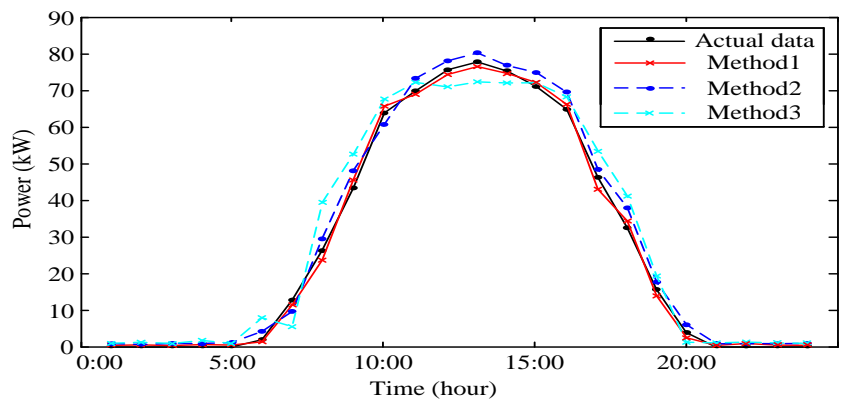

(a) The prediction results in sunny day (Method 1: the hybrid prediction

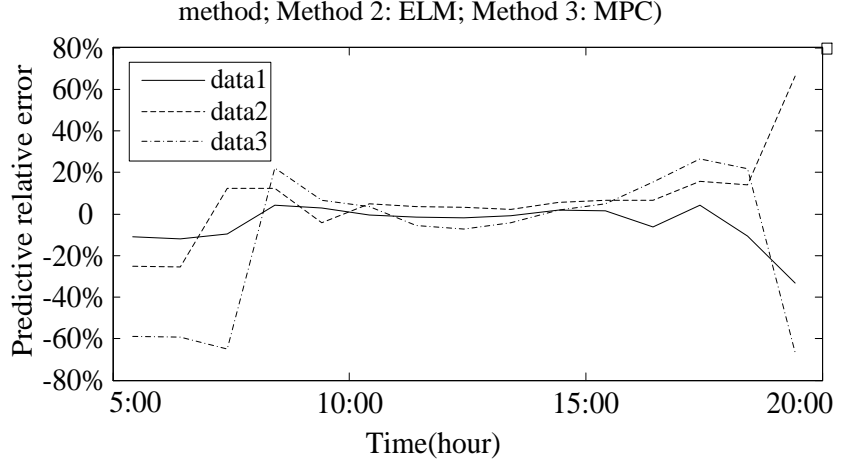

(b) The relative error in sunny days

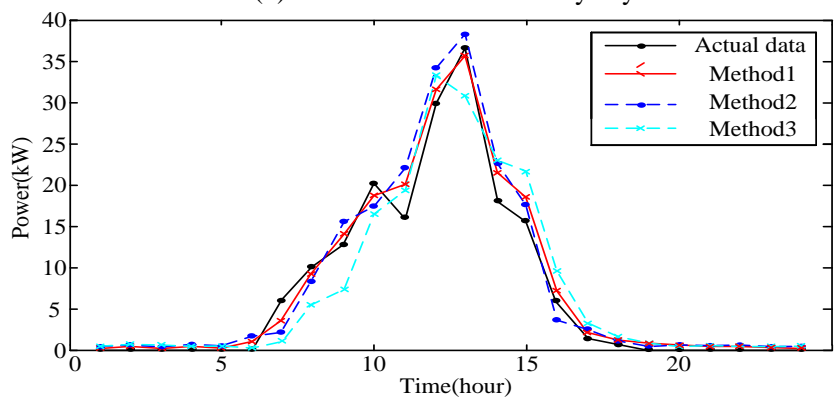

(c) The prediction results in rainy days

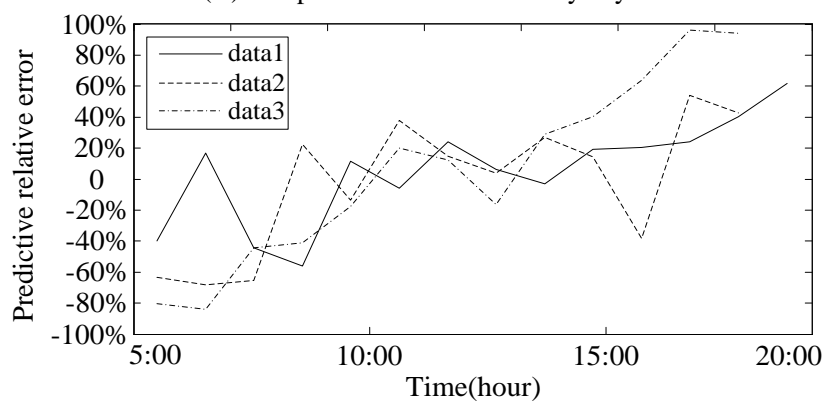

(d) The relative error in rainy days

The simulation results above show that under normal conditions, the prediction accuracy can be improved effectively by using the proposed method. Furthermore, for the PV power generation in uncertain weather conditions, we have also carried out the corresponding simulation study. Note from [40] that PV power generation is mainly related to the length of solar irradiation time, radiation intensity and ambient temperature. Therefore, in this simulation, the historical photovoltaic power generation data in summer and cloudy period in this area (i. e. when the intensity of light is very unstable) is chosen as the testing data. Moreover, these cloudy days are basically around 11:00 to 14:00, and the temper- ature reaches its lowest point at $\mathrm{t}=13: 00$. Then the cloud is gradually cleared. Based on the principle of similar day selection [41], there are 10 similar days of data are selected. In addition, there are 5 days of data randomly selected to train ELMs. After that, one day's data are randomly selected from the remaining five days to test the effect of training. In addition, to demonstrate the effectiveness of the proposed method, the relative errors of these three prediction methods in 24 hours are presented. The simulation results are shown in Fig. 9 (e)-(f), where Method ${ }^{*}$ is our method, while Method 2 is *** Method 3 is ${ }^{* * *}$; and Data 1, Data 2, and Date 3 are $* * * * * *$, respectively.

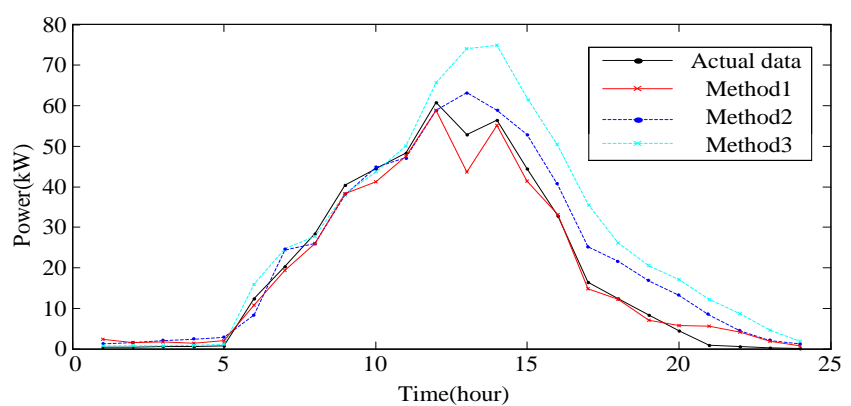

(e ) The prediction results in uncertain weather

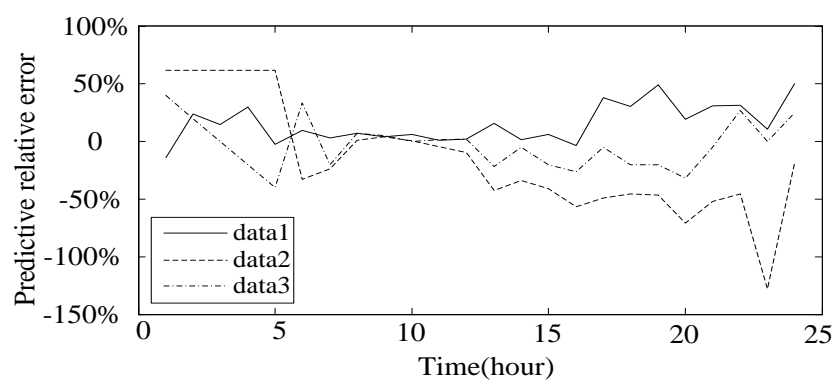

(f) The relative error in uncertain weather

As shown in Fig. 9 (e)-(f), due to the uncertainty of the weather, the regularity of the historical power generation data is not obvious. The variation trend of these data is as follows: Between $t=1: 00$ and $t=12$ : 00 , the electricity generation gradually increases. However, between $t=13: 00$ and $t=14: 00$, there will be a temporary decline and then a rise. After $t=14: 00$, the electricity generation will continue to decline. Moreover, since the control structure of the DER itself remains unchanged, the predicted data output from the MPC is still similar to the input data at the initial iteration time. That is, if the data used for initial iteration is not in consistent well with the data used for testing, it is impossible to obtain the data with high prediction accuracy. When the single ELM is chosen as the prediction method, due to the randomness of the weights between the input and hidden layers, the predicted data are still quite different from the actual data. 
Through these observations, it can be concluded that this new method combines the advantages of MPC and ELM, and its output data are the closest to the actual data.

Table IX Forecast result evaluation and contrast for the three methods

\begin{tabular}{ccccc}
\hline Day type & Error type & Method 1 & Method 2 & Method 3 \\
\hline \multirow{2}{*}{ Sunny day } & MAPE(\%) & 3.6951 & 6.2550 & 12.8474 \\
& RMSE & 3.9025 & 7.1836 & 15.0353 \\
\hline \multirow{2}{*}{ Rainy day } & MAPE(\%) & 6.7247 & 11.5603 & 23.6142 \\
\multirow{2}{*}{ Uncertain } & RMSE & 9.0462 & 15.4818 & 27.9330 \\
day & RAPE(\%) & 3.3383 & 19.2847 & 39.2578 \\
\hline
\end{tabular}

From Table IX, it is found that in sunny days, the MAPE and RMSE of our new method are $40 \%$ and $45 \%$ lower than that of Method 2, respectively, and 71\% and 74\% lower than Method 3. In rainy days, the MAPE and RMSE of the new method are both $41 \%$ lower than Method 2, and are $71 \%$ and $67 \%$ lower than Method 3, respectively. In uncertain days, the MAPE and RMSE of the new method are about $82 \%$ and $91 \%$ lower than Method 2, and are $80 \%$ and $88 \%$ lower than Method 3, respectively.

VI. 2. Verification of vulnerability assessment

In this subsection, the four DERs in Fig. 1 are used to carry out the simulation. The physical and cyber vulnerability value on these nodes are given in Table X XIII.

Table X Physical vulnerability of CPNs

\begin{tabular}{|c|c|c|c|c|c|}
\hline \multirow{2}{*}{$\begin{array}{c}\text { Vulnerability } \\
\text { indexes }\end{array}$} & \multicolumn{5}{|c|}{ MG } \\
\hline & \multicolumn{2}{|c|}{ Line 1} & Line 2 & Line 3 & Line 4 \\
\hline$d_{L q(l)}$ & \multicolumn{2}{|c|}{0.612} & 0.613 & 0.614 & 0.615 \\
\hline$I_{1, L q(l)}$ & \multicolumn{2}{|c|}{0.250} & 0.250 & 0.250 & 0.250 \\
\hline$I_{2, L q(l)}$ & \multicolumn{2}{|c|}{0.250} & 0.250 & 0.250 & 0.250 \\
\hline$\lambda_{L q(l)}$ & \multicolumn{2}{|c|}{1.000} & 1.000 & 1.000 & 1.000 \\
\hline Vulnerability value & \multicolumn{2}{|c|}{0.0383} & 0.0383 & 0.0383 & 0.0384 \\
\hline \multicolumn{6}{|c|}{ Table XI Cyber vulnerability of CPNs } \\
\hline \multirow{2}{*}{\multicolumn{2}{|c|}{$\begin{array}{l}\text { Vulnerability } \\
\text { indexes }\end{array}$}} & \multicolumn{4}{|c|}{ MG } \\
\hline & & Node 1 & Node 2 & Node 3 & Node 4 \\
\hline \multicolumn{2}{|c|}{ Node importance degree } & 1 & 3 & 5 & 5 \\
\hline \multicolumn{2}{|c|}{ The number of failures } & 1 & 3 & 5 & 3 \\
\hline \multicolumn{2}{|c|}{ Protective measures } & 1 & 1 & 3 & 3 \\
\hline
\end{tabular}

\begin{tabular}{c|cccc}
\hline \multicolumn{5}{c}{ Table XII Vulnerability indexes of CPNs after dimensionless } \\
\cline { 2 - 5 } $\begin{array}{c}\text { Vulnerability } \\
\text { indexes }\end{array}$ & \multicolumn{4}{c}{ NG } \\
\cline { 2 - 5 } Physical vulnerability index $\left(\xi_{1}\right)$ & 0.000 & 0.000 & 0.000 & 1.000 \\
Node importance degree $\left(\xi_{2}\right)$ & 0.000 & 0.500 & 1.000 & 1.000 \\
The number of failures $\left(\xi_{3}\right)$ & 0.000 & 0.500 & 1.000 & 0.500 \\
Protective measures $\left(\xi_{4}\right)$ & 0.000 & 0.000 & 1.000 & 1.000 \\
\hline
\end{tabular}

Based on the above Table IV $\sim \mathrm{VI}$, the weighting parameters in Table II can be obtained as follows: $\psi_{1}=0.503 ; \psi_{2}=0.120$; $\psi_{3}=0.126 ; \psi_{4}=0.251$. The prospect vulnerability of each node can be expressed in Table VII.

Table XIII Obtained prospect vulnerability of CPNs

\begin{tabular}{c|cccc}
\hline Vulnerability & \multicolumn{4}{c}{ MG } \\
\cline { 2 - 5 } indexes & Node 1 & Node 2 & Node 3 & Node 4 \\
\hline $\mathrm{v}\left(\xi_{1}\right)$ & 0.000 & 0.000 & 0.000 & 1.000 \\
$\mathrm{v}\left(\xi_{2}\right)$ & 0.000 & 0.543 & 1.000 & 1.000 \\
$\mathrm{v}\left(\xi_{3}\right)$ & 0.000 & 0.543 & 1.000 & 0.543 \\
$\mathrm{v}\left(\xi_{4}\right)$ & 0.000 & 0.000 & 1.000 & 1.000 \\
Prospect value & 0.000 & 0.134 & 0.497 & 0.942 \\
\hline
\end{tabular}

In order to verify the effectiveness of vulnerability assessment more intuitively, the comparison on node vulnerability is shown in Fig. 10. It can be seen from this figure that the physical vulnerability of CPN 4 is the highest, and the cyber vulnerability of CPN 3 is the highest. Moreover, the other three CPNs have the same physical vulnerability. After using cyber-physical vulnerability assessment, the prospect vulnerability of CPN 4 remains the highest. However, the prospect vulnerabilities of CPN 3, CPN 2 and CPN 1 decrease in turn. Therefore, it is not sufficient to consider only physical vulnerability or cyber vulnerability alone, and a combination of the two is needed.

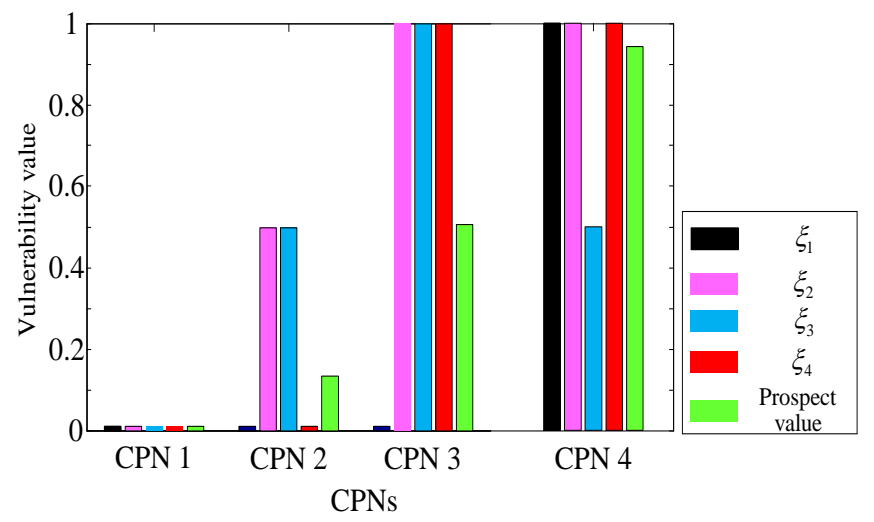

Fig. 10 The prospect vulnerability of $\mathrm{CPN} s$

According to [42, 43], sensitivity analysis is an analytical technique to study the influence of the change of relevant parameters on an evaluation result from the perspective of quantitative analysis. To better demonstrate the impact of a change in an index on the prospect vulnerability of CPNs, the sensitivity coefficient is used herein. The sensitivity coefficient provides the ratio between the change rate of each index and the change rate of the evaluation result, which can indicate the sensitivity of the evaluation result to a certain extent. For an index $F$,

to be evaluated in a function or result $A$, the formula for the sensitivity coefficient is $S_{\text {Sens. } F}=\frac{\Delta A / A}{\Delta F / F}$

where $\Delta A$ is the variations in evaluation results; and $\Delta F$ is the variations in $F$. Based on the theory above, the sensitivity of the four indicators to the prospect vulnerability of CPNs can be analyzed. The specific analysis process is divided into two steps: 1 . For each of the CPN, a sensitivity analysis is performed on each of the indicators, respectively. The variation of the indexes is selected as $10 \%$; 2. For each CPN, the sensitivity coefficients corresponding to each index change are calculated according to the result of the vulnerability change caused by each index change. After completing the above procedures, the following Fig. 11 can be obtained. 


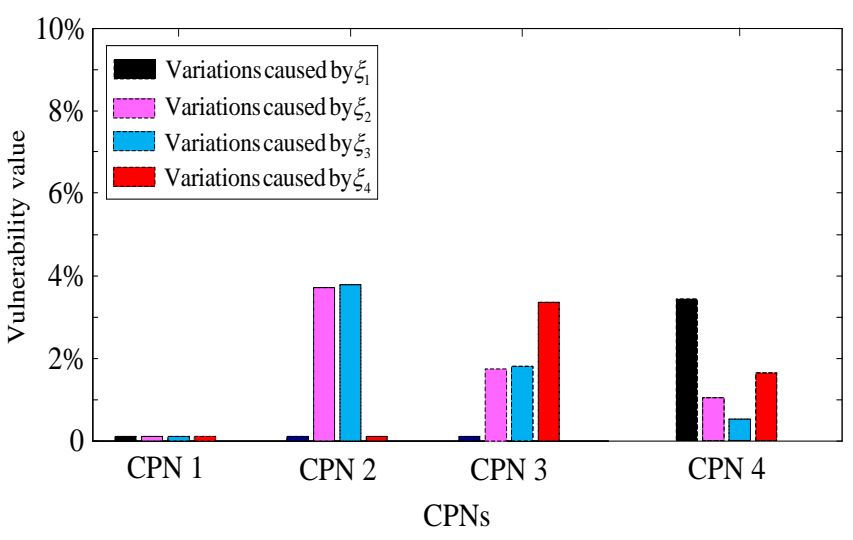

Fig. 11. The variations of prospect value caused by each index

As can be seen from Fig. 11, the impact of the four vulnerability indicators on the prospect vulnerability for CPN 1 is basically 0 . For CPN 2, the prospect vulnerability is mainly affected by the second and third indicators. When the two indicators change by $10 \%$, the foreground values change by $3.609 \%$ and $3.767 \%$, and the impact of the remaining two indicators on the prospect value is also almost 0 . For CPN 3, the prospect value is mainly affected by the latter three indicators. In the above sensitivity analysis for CPN 1, CPN 2 and CPN 3, the changes of prospect values are $1.814 \%$ and $1871 \%$ and $3.419 \%$, respectively. For CPN 4, the vulnerability is mainly affected by the first indicator, and the change of prospect value is $3.428 \%$. The corresponding changes of the other three indexes are almost $0.946 \%$ and $0.536 \%$ and $1.804 \%$, respectively. In addition, to better analyze the impact of each index on the prospect vulnerability of CPNs, the sensitivity coefficients are calculated as follows.

\begin{tabular}{|c|c|c|c|c|}
\multicolumn{5}{r|}{ Table XIV } \\
\hline$S_{\text {Sens }}$ & $S_{\text {Sens. } \xi_{1}}$ & $S_{\text {Sens. } \xi_{2}}$ & $S_{\text {Sens. } \xi_{3}}$ & $S_{\text {Sens. } \xi_{4}}$ \\
\hline CPN 1 & 0.000 & 0.000 & 0.000 & 0.000 \\
\hline CPN 2 & 0.000 & 0.361 & 0.377 & 0.000 \\
\hline CPN 3 & 0.000 & 0.181 & 0.187 & 0.342 \\
\hline CPN 4 & 0.343 & 0.095 & 0.054 & 0.180 \\
\hline
\end{tabular}

To summarize, the sensitivity of CPN 1 to these four categories of indicators is the least. The prospect values of CPN 2 and CPN 3 are mainly affected by the latter three indexes. The prospect value of CPN 4 is mainly affected by the first index.

VI. 3. Verification of EMS

A typical summer day in northern China is selected for the case study. The predicted power data of WT, PV, net load and total load are illustrated in Fig. 12, where net load refers to the difference between the total load and the DER generation.

In this area, the changes of PV, WT and load in 24 hours (one day) are shown in this figure. Solar irradiance in a day follows a regular pattern, i.e., it is the highest in the noon while very low in the early morning and late afternoon. This also makes the PV power generation regular. However, because of the irregularity of wind speed and direction in $24 \mathrm{~h}$, WT power generation is usually not regular. This also verifies the volatility of renewable power generation units. Furthermore, it is found that this area has higher electric load between 8: 00 and 16: 00, and very low demand between 0:00-4:00. It also shows that PV and WT generation alone cannot meet load requirement at particularly the time period 0:00-8:00, and the energy management system needs to use resources from energy storage and controllable distributed generations to meet the load requirement, where flexible load and interruptible load are also properly controlled for the economic operation of the MG.

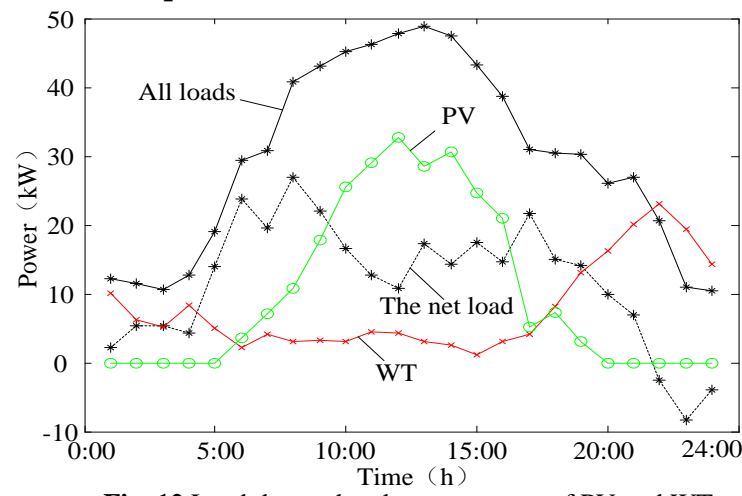

Fig. 12 Load demand and output power of PV and WT

Fig. 13 shows the power consumption data of fixed, interruptible, and flexible loads during the day.

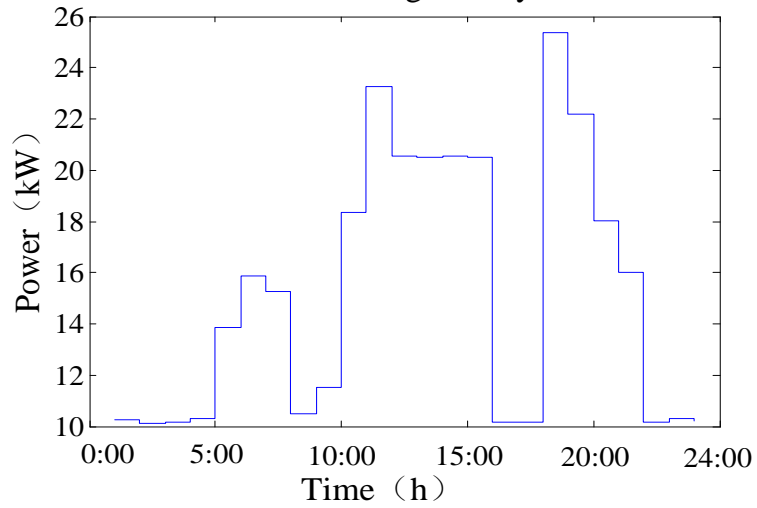

(a) Fixed load 


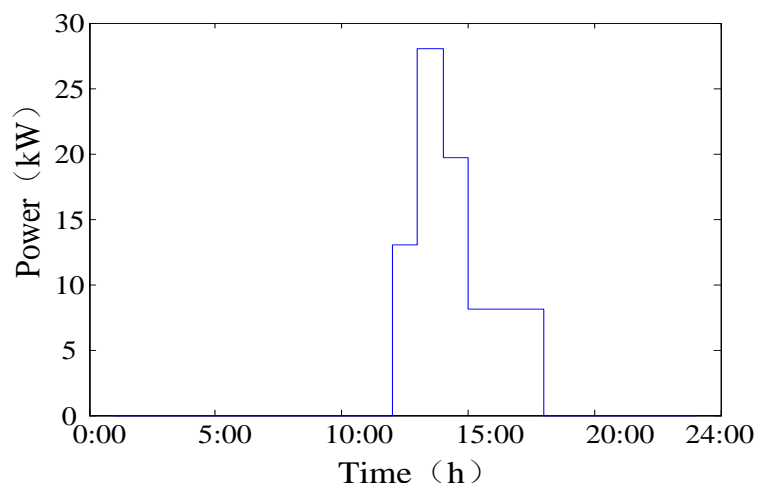

(b) interruptible load

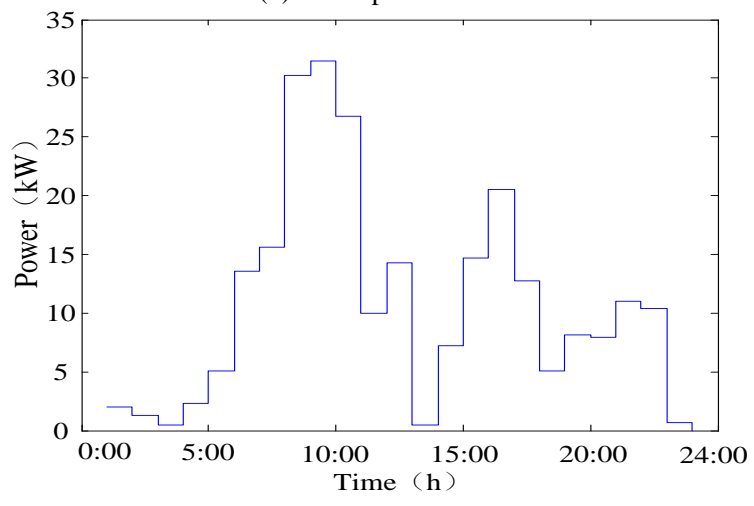

(c) Flexible load

Fig. 13 Power of the fixed and interruptible and flexible load

As shown in Fig. 13 (a), the peak of fixed load mainly happens during 10:00- 16:00 and 18:00-22:00. From Fig. 13 (b), the peak of interruptible load occurs during 12: 00-18: 00, while Fig. 13 (c) shows that the peak of flexible load mainly occurs at 8:00-10:00 or 15:00-17:00. Fig. 14 shows the optimized flexible load and the corresponding net load curve. As can be seen from Fig. 14 (a), the flexible load is shifted from peak to the standard and off-peak periods.

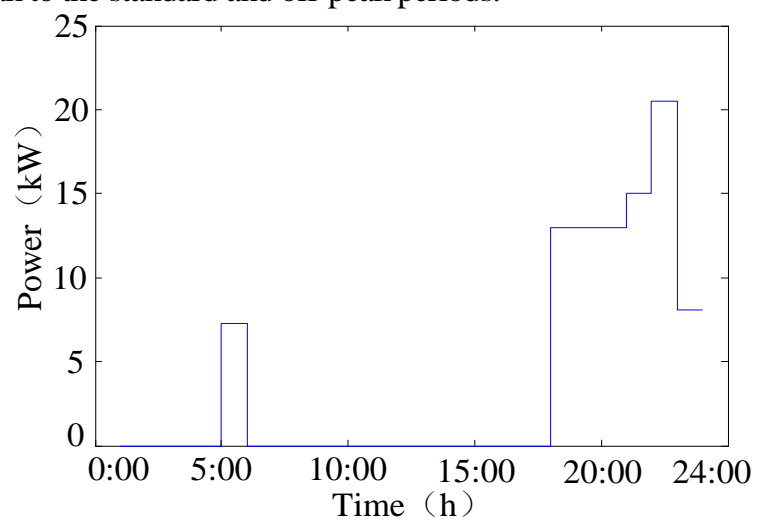

(a) Flexible load

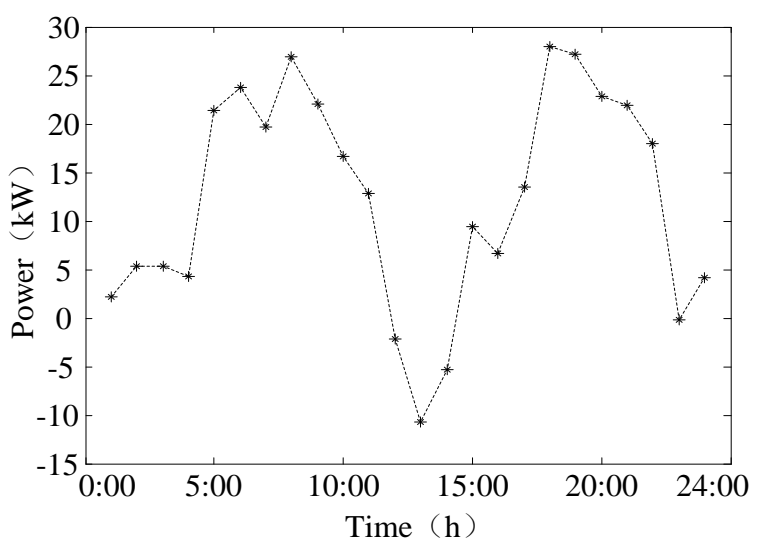

(b) The net load
Fig. 14 Optimization results of flexible load and net load

The optimized power output flows of energy storage unit, DERs (MT and FC) and the main grid are shown in Fig. 15. According to the optimized value of storage unit in Fig. 15 (a), the unit is in discharging status at 5:00-8:00 and 21:00-22:00. Compared with the net load curve in Fig. 15 (b), the discharging period of the energy storage is exactly the peak period of the net load, which helps to reduce high electricity cost at peak periods. It can be observed from Fig. 15 (b) that the output of controllable DERs is relatively stable. However, the fluctuation in output power of the main grid is relatively large, owning primarily to the high generation from the PV in the noon.

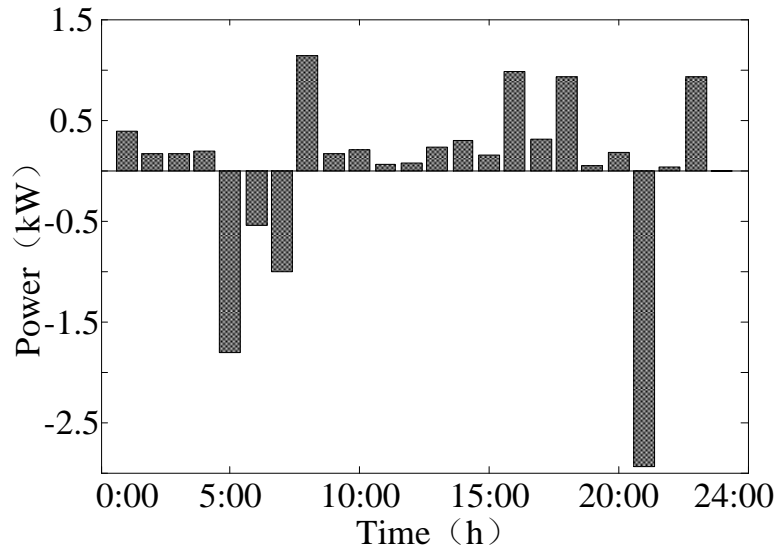

(a) The planned output power of energy storage unit

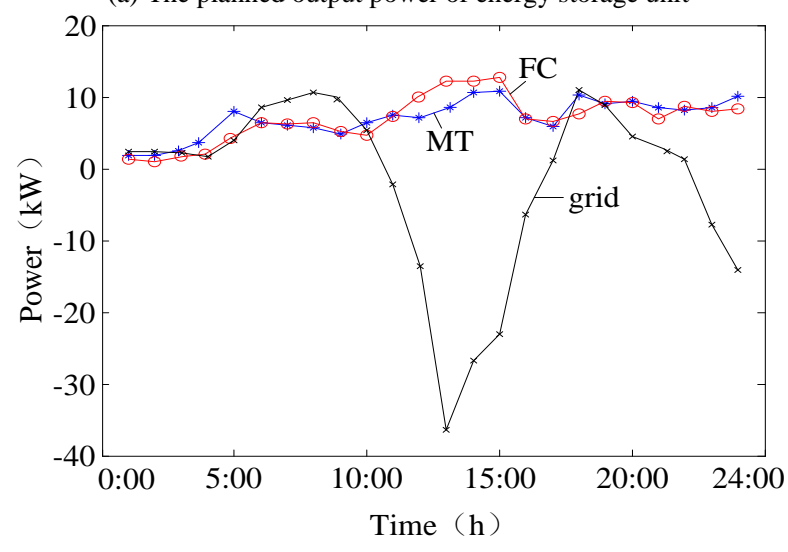

(b) Power outputs of controllable DERs and main grid

Fig. 15 Optimization results of rolling optimization

Fig. 16 and Fig. 17 show the cost before and after optimization by using ordinary PSO and the improved PSO, respectively. 


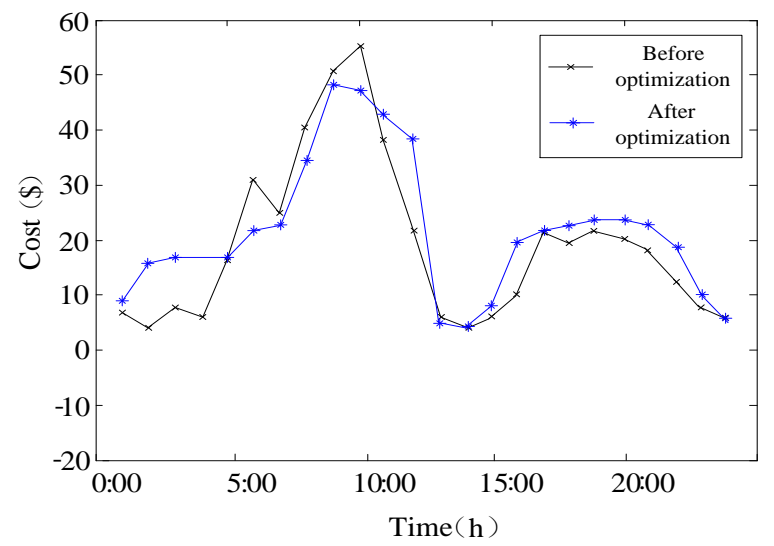

Fig. 16 Cost comparison by ordinary PSO

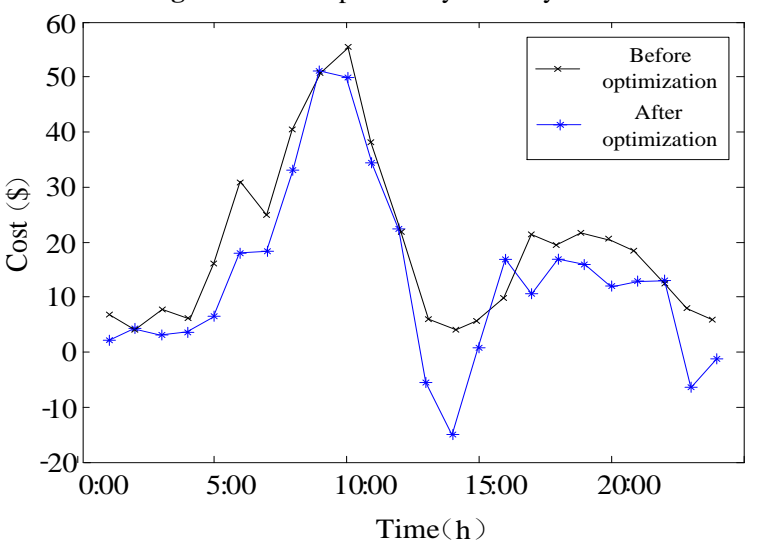

Fig. 17 Cost comparison by improved PSO

Based on the above two figures, it is found that the overall cost of 24 hours before optimization is about \$538.1; and it changes to $\$ 522.6$ by using the ordinary PSO algorithm, and $\$ 293.5$ by the improved PSO. This optimized cost by the improved PSO is about $54.5438 \%$ of the original cost before optimization. The DSR scheme obtained by ordinary PSO algorithm is obviously more costly than that obtained by improved PSO algorithm. This also shows that the ordinary PSO algorithm is more likely to fall into the local optimal solution.

The values of the objective function before optimization are all greater than zero, and the MG has only expenditures. However, after optimization, the total expenditure cost of MG is obviously reduced and the system has positive savings.

VI. 4. Verification of neighbor prediction

In this subsection, the predicted generation data of DER1 at 5:00 are assumed to be lost during the transmission to the EMS part. From subsection VI. 2, it is found that the interrupted data can be transmitted by the input channel of DER 2 because that the vulnerability of CPN2 is minimum. The corresponding optimized results are shown in Fig. 18.

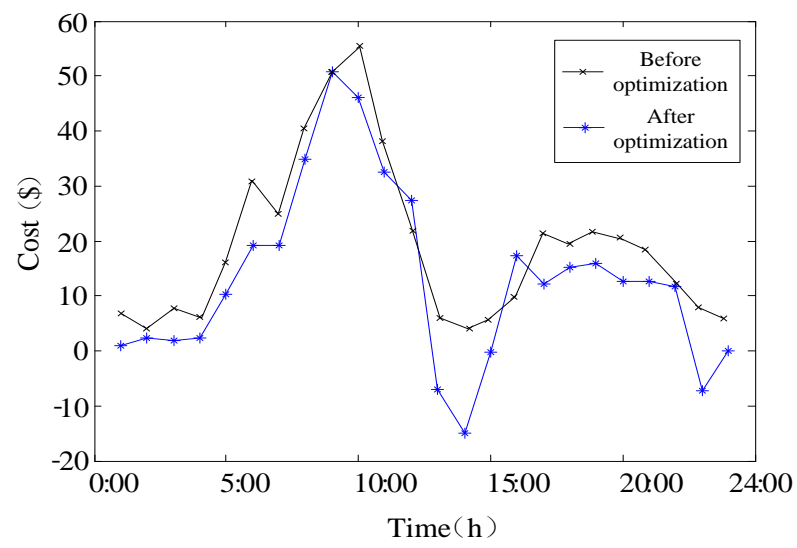

Fig. 18 Cost comparison under interrupted data transmission

As shown in Fig. 18, the solution can still optimize normally before 5:00. But at 5: 00, the predicted data of DER1 is interrupted. To maintain the balance of power supply and demand, the cost has been increased. At the next moment, the optimization process can be carried out normally through neighbor prediction. Moreover, it is found that the overall cost of MG is reduced significantly. It also illustrates that the neighbor prediction method can effectively overcome the problem of data interruption.

\section{Conclusion}

To effectively manage the data transmission interruption problem in MG operations, a hybrid prediction-based energy management method is proposed in this paper. Interruptible, flexible and fixed loads are considered in the demand response of MG energy management, where distributed energy resources and energy storage are also considered within the MG. , Based on vulnerability assessment and prospective theory, a neighbor prediction method is proposed to solve the interruption problem in the process of transferring predicted data to the energy management system. In the end, an optimization model considering demand side response is established, and an improved PSO algorithm is proposed to solve the model effectively.

Through the simulation analysis, it can found that the proposed hybrid prediction method can reduce at least $40 \%$ prediction error compared with method of using MPC or ELM alone. The proposed optimal energy management solution can effectively schedule the output of DER generations and control the load, and the optimized cost has been reduced to $54.5438 \%$ of the original cost. When one hour's data are lost, the energy management system still works properly, and it can save $* * \%$ cost than the original case before optimization. Therefore, these case studies verify that the proposed energy management model works very well under both normal conditions and data interruptions. As a future work, different types of demand response programs and the impact of electric vehicles will be investigated to obtain the optimal energy management plans for the microgrid.

\section{Acknowledgment}

This work is supported by the Natural Science Foundation of Hebei Province (No. F2016203507), the National Natural Science Foundation of China (No. 61873235, 61473246) and Beijing Environment Foundation for Young Talents.

\section{References}


[1] Mahmoud M S , Azher Hussain S , Abido M A . Modeling and control of microgrid: An overview. Journal of the Franklin Institute, 2014, 351(5):2822-2859..

[2] Xu, Y L, Shen X W. Optimal Control Based Energy Management of Multiple Energy Storage Systems in a Microgrid. IEEE Access, 2018, 6: 32925-32934.

[3] Nayanatara C, Baskaran J, Kothari D P. Hybrid optimization implemented for distributed generation parameters in a power system network. International Journal of Electrical Power \& Energy Systems, 2016, 78: 690-699.

[4] He L C, Lu Z G, et al. Low-carbon economic dispatch for electricity and natural gas systems considering carbon capture systems and power-to-gas. Applied Energy, 2018, 224: 357-370.

[5] Zhang Z, Dou C, Yue D, et al. A Decentralized Control Method for Frequency Restoration and Accurate Reactive Power Sharing in Islanded Microgrids. Journal of the Franklin Institute. 2018. doi: 10.1016/j.jfranklin.2018.09.024.

[6] Olivares D E, Canizares C A, Kazerani M. A Centralized Energy Management System for Isolated Microgrids. IEEE Transactions on Smart Grid, 2014, 5(4):1864-1875.

[7] Pourmousavi S A, Nehrir M H, Sharma R K. Multi-Timescale Power Management for Islanded Microgrids Including Storage and Demand Response. IEEE Transactions on Smart Grid, 2015, 6(3):1185-1195.

[8] Li J, Liu Y, Wu L. Optimal Operation for Community Based Multi-Party Microgrid in Grid-Connected and Islanded Modes. IEEE Transactions on Smart Grid, 2018, 9 (2): 756-765.

[9] M.S. Mahmoud, M.S.U Rahman, Event triggered of microgrid control with communication and control optimization. Journal of the Franklin Institute. 2016. 353 (16): 4114-4132.

[10] Solanki B V, Bhattacharya K, Cañizares C A. A Sustainable Energy Management System for Isolated Microgrids. IEEE Transactions on Sustainable Energy, 2017, 8(4):1507-1517.

[11] Palma-behnke R, Benavides C, Lanas F, et al. A Microgrid Energy Management System Based on the Rolling Horizon Strategy. IEEE Transactions on Smart Grid, 2013, 4(2):996-1006.

[12] Pourmousavi S A, Nehrir M H, Sharma R K. Multi-Timescale Power Management for Islanded Microgrids including Storage and Demand Response. IEEE Transactions on Smart Grid, 2015, 6(3): 1185-1195.

[13] Tsikalakis A G Hatziargyriou N D. Centralized Control for Optimizing Microgrids Operation. IEEE Transactions on Energy Conversion, 2008, 23(1): 241-248.

[14] Sechilariu M, Wang B, Locment F. Building Integrated Photovoltaic System with Energy Storage and Smart Grid Communication. IEEE Transactions on Industrial Electronics, 2013, 60(4): 1607-1618.

[15] Taylor J A, Callaway D S, Poolla K . Competitive Energy Storage in the Presence of Renewables. IEEE Transactions on Power Systems, 2013, 28(2): 985-996.

[[16] Murray G, Alexa S , Jamie W , et al. Differentiating 'the user' in DSR: Developing demand side response in advanced economies. Energy Policy, 2018, 122:176-185.

[17] Rezaee Jordehi A. Optimization of demand response in electric power systems, a review. Renewable and Sustainable Energy Reviews, 2019, 103: 308-319.

[18] Hongbo C, Kaile Z. Industrial power load scheduling considering demand response, Journal of Cleaner Production, 2018, 204: 447-467.

[19] Damian G, Papadopoulos A I , Charalampos P, et al. A systems approach for management of microgrids considering multiple energy carriers, stochastic loads, forecasting and demand side response. Applied Energy, 2018, 226:546-559.

[20] Wang Y, Mao S, and Nelms R M. Distributed online algorithm for optimal real-time energy distribution in the smart grid. IEEE Internet Things Jounal, 2014, 1(1): 70-80.

[21] Chiu T C, Shih Y Y, Pang A C, et al. Optimized Day-Ahead Pricing with Renewable Energy Demand-Side Management for Smart Grids. IEEE Internet of Things Journal, 2017, 4(2): 374-383.
[22] Eksin C, Delic H, Ribeiro A. Demand Response Management in Smart Grids with Heterogeneous Consumer Preferences. IEEE Transactions on Smart Grid, 2015, 6(6): 3082-3094.

[23] Logenthiran T, Srinivasan D, Shun T Z. Demand Side Management in Smart Grid Using Heuristic Optimization. IEEE Transactions on Smart Grid, 2012, 3(3): 1244-1252.

[24] Yao E, Samadi P , Wong V W S , et al. Residential Demand Side Management Under High Penetration of Rooftop Photovoltaic Units. IEEE Transactions on Smart Grid, 2017, 7(3): 1597-1608.

[25] Ye F , Qian Y, Hu R. A Real-Time Information Based Demand-Side Management System in Smart Grid. IEEE Transactions on Parallel \& Distributed Systems, 2016, 27(2): 329-339.

[26] Tushar M H K, Assi C, Maier M. Distributed Real-Time Electricity Allocation Mechanism for Large Residential Microgrid. IEEE Transactions on Smart Grid, 2015, 6(3):1353-1363.

[27] Dou C, Zhang Z, Yue D, et al. Improved droop control based on virtual impedance and virtual power source in low-voltage microgrid. IET-Generation, Transmission \& Distribution, 2017, 11(4):1046-1054.

[28] Xiao L , Mandayam N B , Vincent Poor H. Prospect Theoretic Analysis of Energy Exchange Among Microgrids. IEEE Transactions on Smart Grid, 2015, 6(1):63-72.

[29] Mishra M, Rout P K. Detection and classification of micro-grid faults based on HHT and machine learning techniques. IET-Generation Transmission \& Distribution, 2018, 12(2): 388- 397. [30] Determination of optimal super capacitor-lead-acid battery energy storage capacity for smoothing wind power using empirical mode decomposition and neural network. Electric Power Systems Research, 2015, 127: 323-331.

[31] Huang G B, Zhou H , Ding X , et al. Extreme Learning Machine for Regression and Multiclass Classification. IEEE Transactions on Systems, Man and Cybernetics, Part B Cybernetics, 2012, 42(2):513-529.

[32] Yang Q, Yuan D, Guo X, Zhang B, Zhi C. A Novel Hierarchical Control Strategy for Low-Voltage Islanded Microgrids Based on the Concept of Cyber Physical System. Energies. 2018, 11, 1835. DOI: 10.3390/en11071835

[33] XuY Q, Liu D, Zhang Y. Modeling of wind speed correlation based on entropy weighted fuzzy comprehensive evaluation. Journal of engineering, 2017, (13): 2037-2042.

[34] Bruno S, Dassisti M, Scala M L, et al. Predictive Dispatch Across Time of Hybrid Isolated Power Systems. IEEE Transactions on Sustainable Energy, 2014, 5(3):738-746.

[35] Dou C X, Meng C H, Yue W B, et al. Double-deck optimal schedule of micro-grid based on demand-side response. IET-Renewable Power Generation, 2018, doi: 10.1049/iet-rpg. 2018.5495.

[36] Papadaskalopoulos D , Pudjianto D, Strbac G . Decentralized Coordination of Microgrids With Flexible Demand and Energy Storage. IEEE Transactions on Sustainable Energy, 2014, 5(4):1406-1414.

[37] Dou C X, Zhang B, Yue D, et al. Cyber-Physical Cooperative Control Strategy for Islanded Micro-grid considering with Communication Interruption. International Transactions on Electrical Energy Systems. 2018, doi: 10.1002/ etep. 2695.

[38] Hassan M A, Abido M A. Optimal design of microgrids in autonomous and grid-connected modes using particle swarm optimization. IEEE Transactions on Power Electronics, 2011, 26 (3):755-769.

[39] Sun J , Wu X , Palade V , et al. Convergence analysis and improvements of quantum-behaved particle swarm optimization. Information Sciences, 2012, 193: 81-103.

[40] Eseye A T, Zhang J, Zheng D, et al. A double-stage hierarchical ANFIS model for short-term wind power prediction. IEEE International Conference on Cloud Computing and Big Data Analysis, 2017: 342-349.

[41] Zhang X m, Li Y, Lu S Y, et al. A Solar Time Based Analog Ensemble Method for Regional Solar Power Forecasting. IEEE Transactions on Sustainable Energy, 2019, 10(1): 268-279. 
[42] Moghaddam I N , Salami Z, Easter L. Sensitivity Analysis of an Excitation System in Order to Simplify and Validate Dynamic Model Utilizing Plant Test Data. IEEE Transactions on Industry Applications, 2015, 51(4):3435-3441.

[43] Chen J , Chen J . Stability Analysis and Parameters Optimization of Islanded Microgrid With Both Ideal and Dynamic Constant Power Loads. IEEE Transactions on Industrial Electronics, 2018, 65(4):3263-3274. 